\title{
Long term variations of galactic cosmic radiation on board the International Space Station, on the Moon and on the surface of Mars
} \author{
Nathan A. Schwadron ${ }^{4}$, Harlan E. Spence ${ }^{4}$, Donald M. Hassler ${ }^{5}$, Bent Ehresmann ${ }^{5}$, \\ and Robert F. Wimmer-Schweingruber ${ }^{2}$ \\ ${ }^{1}$ German Aerospace Center (DLR), Institute of Aerospace Medicine, 51147 Cologne, Germany \\ ${ }^{2}$ Christian Albrechts University (CAU), 24118 Kiel, Germany \\ ${ }^{3}$ Leidos, Houston, 77058 TX, USA \\ ${ }^{4}$ University of New Hampshire, Durham, 03824 NH, USA \\ ${ }^{5}$ Southwest Research Institute, Boulder, 80302 CO, USA
}

Thomas Berger ${ }^{1, *}$, Daniel Matthiä ${ }^{1}$, Sönke Burmeister ${ }^{2}$, Cary Zeitlin ${ }^{3}$, Ryan Rios ${ }^{3}$, Nicholas Stoffle ${ }^{3}$,

Received 9 March 2020 / Accepted 16 June 2020

\begin{abstract}
The radiation environment in free space and the related radiation exposure is seen as one of the main health detriments for future long-duration human exploration missions beyond Low Earth Orbit (LEO). The steady flux of energetic particles in the galactic cosmic radiation (GCR) produces a low dose-rate radiation exposure, which is heavily influenced by several factors including the solar cycle, the presence of an atmosphere, relevant magnetic fields (as on Earth) and of course by the relevant spacecraft shielding. Investigations of the GCR variations over the course of a solar cycle provide valuable data for exploration mission planning and for the determination of the radiation load received due to the GCR environment. Within the current work these investigations have been performed applying three datasets generated on board the International Space Station (ISS) with the DOSTEL instruments in the frame of the DOSIS and DOSIS-3D projects, with the CRaTER instrument in a Moon orbit and with the MSL-RAD instrument on the way to and on the surface of Mars. To derive GCR dose contributions on board the ISS two procedures have been developed separating the contributions from GCR from passing's through the South Atlantic Anomaly (SAA), as well as ways to extrapolate the GCR dose measured on board the ISS to free space based on various ranges of the McIlwain $L$-shell parameter. At the end we provide a dataset spanning the timeframe for GCR measurements on the ISS (2009-2011 \& 2012-2019), Moon (2009-2019) and Mars (2012-2019), thereby covering the time span from the deep minimum of solar cycle 23 , the ascending phase and maximum of solar cycle 24 , and the descending phase of cycle 24 , which is ongoing at the time of this writing.
\end{abstract}

Keywords: International Space Station / Moon / Mars / Galactic cosmic radiation / Solar particle events

\section{Introduction}

The space radiation environment and its impact on humans have been seen as one of the main challenges for long duration human space missions. This is of particular importance for the currently planned exploration missions to the Moon, near Earth asteroids and in the long term Mars (Durante \& Cucinotta, 2011). The radiation environment in space is composed mostly of galactic cosmic radiation (GCR), which - modulated by solar activity - provides the overall background radiation environment in free space (FS). For long duration missions GCR will be the primary source of stochastic radiation effects resulting

\footnotetext{
*Corresponding author: thomas. berger@dlr.de
}

in additional risk of exposure induced cancer (REIC). Another source of radiation are the protons that are accelerated during a solar particle event (SPE). Astronauts on board the International Space Station (ISS) are protected against high doses from solar energetic protons (SEP) as a result of being shielded by the geomagnetic field for much of its trajectory. This will not be the case for trajectories in free space or on the surface of planetary bodies without a magnetic field and/or substantial atmosphere, such as the Moon and Mars.

GCR is the main background radiation in free space as well as on the surface of other celestial bodies that lack thick atmospheres. The GCR environment is influenced by various parameters. The main driver of changes in the GCR environment in free space is the Sun due to the 11-year solar cycle, which 
causes variations in the strength and structure of the interplanetary magnetic field. This in turn leads to the highest GCR rates during solar minimum and the lowest during solar maximum conditions. For Mars an additional factor is the interaction of the GCR environment with the atmosphere leading to the creation of secondary particles (neutrons, light ions, etc.) that can reach the surface and increase the complexity of the radiation field. Depending on the relevant atmospheric depths the GCR environment varies. The situation is different for LEO due to the fact that Earth has a magnetic field which leads to strong dependence of the GCR dose rates on latitude and longitude. In addition the interaction of GCR with a spacecraft itself leads to a secondary (GCR) induced radiation field inside this spacecraft. The variations of GCR have been measured indirectly on Earth for several decades using the neutron monitor (NM) network.

Within this work we are aiming to compare long term measurements of the GCR environment performed with radiation detector systems on board the International Space Station (ISS), in the orbit of the Moon, on the cruise phase to Mars and on the surface of Mars, thereby showing how the GCR dose is influenced by the various parameters as given above. With this we are covering the time span from the deep minimum of solar cycle 23 , the ascending phase and maximum of solar cycle 24 , and the descending phase of cycle 24 , which is ongoing at the time of this writing. Of special importance is that this is the first time that long continuous dose rate measurements in LEO (2009-2011 \& 2012-2019) are compared to similarly long-term continuous data from the vicinity of the Moon (2009-2019) and the surface of Mars (2012-2019).

\section{Instruments used within this study}

Within this study three different instruments and their data are used for the comparison of GCR dose values over a relevant timeframe (see Sect. 2.4). This instrument set comprises the DOSIS \& DOSIS 3D DOSTEL detectors measuring inside the Columbus Laboratory of the ISS (Berger et al., 2016, 2017, 2018), the CRaTER instrument circling around the Moon on board the Lunar Reconnaissance Orbiter (LRO) spacecraft (Spence et al., 2010) and the MSL-RAD instrument (Hassler et al., 2012) measuring on the way to Mars (Zeitlin et al., 2013) and on the surface of Mars as part of the Mars Science Laboratory (MSL) Curiosity mission since 2012 (Hassler et al., 2014).

All the instruments have been described in detail in the relevant references, therefore we are going to provide only a short description of the instruments and the relevant quantities measured. It has to be noted that all absorbed dose values within this manuscript are given in absorbed dose in $\mathrm{Si}$. This also avoids any issues with using different conversion coefficients between the absorbed dose in $\mathrm{Si}$ and the absorbed dose in $\mathrm{H}_{2} \mathrm{O}$.

\subsection{The DOSIS 3D-DOSTEL instruments}

The DOSTEL instrument family has established its pedigree with various space missions on board space shuttle (STS) flights, the space station Mir and the ISS. In the framework of the DOSIS (2009-2011) and the DOSIS 3D (Dose Distribution Inside the
ISS 3D) project (since 2012 and ongoing) (Berger et al., 2016, 2017, 2018), two DOSTEL units are positioned beneath the European Physiology Module (EPM) Rack for long term dose monitoring on board the European Columbus module. For this experiment, the DOSTEL units (D-1 and D-2) are connected to the DOSTEL data and power unit (DDPU). The DDPU supplies the two instruments with power, stores the data from the instruments on an SD memory card and provides the electrical (data/ power) interface to the Columbus module. The DOSTEL units are located together with the DDPU inside a NOMEX pouch, the DOSIS-MAIN-BOX, so that the viewing direction of the telescope of DOSTEL-1 is in the forward (ISS flight) direction, whereas DOSTEL-2 is directed perpendicular (starboard/port) to the ISS flight direction (see Fig. 1 in Berger et al., 2017).

Each DOSTEL consists of two passivated implanted planar silicon (PIPS) detectors (D1 and D2) each with a thickness of $315 \mu \mathrm{m}$ and an active area of $6.93 \mathrm{~cm}^{2}$. These detectors are arranged in a telescope geometry mounted at a distance of $15 \mathrm{~mm}$ thereby yielding a telescope with an opening angle of approximately $120^{\circ}$ and a geometric factor for isotropic irradiation from one side of $824 \mathrm{~mm}^{2} \mathrm{sr}$, if coincident hits in both detectors are required. Using this coincidence requirement the limited path length in the detectors allows deriving information about the linear energy transfer (LET) spectra from the measured energy depositions. The DOSTEL units also acquire count rates and dose rates of radiation hitting the single detectors ("dose measurement"). The measured dose rate results from the sum of the energy depositions per unit time. The detection ranges for the measured energy depositions are 0.069-165 MeV for the D1 detector and of $0.048-66 \mathrm{MeV}$ for the D2 detector, both ranges are for energy depositions in $\mathrm{Si}$. During the DOSIS project the instruments measured from 2009 to 2011 (DOSTEL-1 only until 2010). For DOSIS 3D (D3D) the hardware was launched with Soyuz 30S on 15 May 2012, installed beneath the EPM rack and started measurements on 21 May 2012. The scientific and housekeeping data are downloaded by the EPM rack via Ethernet connection nominally every four weeks. As of this writing, the instruments have measured for over eight years at this dedicated position inside Columbus. Further detailed description of the instruments as well as all relevant information about calibration as well as detailed information about data products is given in Berger et al. (2016, 2017, 2018).

\subsubsection{DOSIS 3D-DOSTEL: data products}

During their operation on orbit, the two DOSTEL units (D-1/D-2) independently measure the particle count- and absorbed dose rates in there single DOSTEL detectors (D1/ D2). Count rate and absorbed dose rate information is stored every $100 \mathrm{~s}$ for GCR-like contributions and a count rate trigger decreases the integration time to $20 \mathrm{~s}$ for SAA passes.

\subsection{The CRaTER instrument}

CRaTER (Spence et al., 2010) is a particle telescope composed of six silicon detectors, flying aboard the LRO spacecraft. LRO reached lunar orbit in July 2009, and the spacecraft's orbit was circularized in September 2009, and CRaTER has been recording GCR (see for example Zeitlin et al., 2019a) and SEP (see for example Schwadron et al., 2018) fluxes with very few interruptions since then. CRaTER data cover the time 
span from the deep cycle 23 solar minimum, the ascending phase and maximum of cycle 24 , and the descending phase of cycle 24, which is ongoing at the time of this writing. The design of the CRaTER instrument is unusual in that much of the volume of the telescope volume is occupied by passive material, A-150 tissue-equivalent plastic (TEP). The six silicon diodes are arranged in three pairs, each consisting of one thin $(148 \mu \mathrm{m})$ and one thick $(1000 \mu \mathrm{m})$ planar detector. The telescope's boresight is usually pointed along the zenith/nadir axis, with occasional brief exceptions when the spacecraft is rotated to allow LRO's cameras to image various lunar features from different angles. The first detector pair of the CRaTER telescope (detectors D1/D2) is under minimal shielding - enough to stop solar wind ions but not GCRs or SEPs - and measures the freespace environment; a $6 \mathrm{~g} / \mathrm{cm}^{2}$ piece of TEP is located between the first and second (D3/D4) pairs, and an additional piece of $\mathrm{TEP}$, this one $3 \mathrm{~g} / \mathrm{cm}^{2}$ in depth, is located between the second and third pairs (D5/D6). The sensitivity of the thick detectors (D2, D4, and D6) extends to minimum-ionizing single-charged particles, but their pulse-height readout electronics saturate at LET in Si of $88 \mathrm{keV} / \mu \mathrm{m}$, corresponding roughly to highly relativistic sulfur (charge 16). The thin detectors (D1, D3, and D5) do not efficiently record particles with LET in Si below about $7 \mathrm{keV} / \mu \mathrm{m}$, but can measure up to $2000 \mathrm{keV} / \mu \mathrm{m}$ without saturating.

\subsubsection{CRaTER: data products}

In the following we only focus on CRaTER data using the combination of the D1 and the D2 spectra $\left(\mathrm{CRaTER}_{\mathrm{D} 1 / \mathrm{D} 2}\right)$. The limited dynamic ranges of both D1 and D2 make it necessary to combine the two measurements in order to report the dose over the full LET spectrum. Conceptually, one can think of the D1 measurement as the dose excluding the lightest ions, whereas the dose measured by D2 excludes the heaviest ions. There is considerable overlap of the two LET spectra, and the choice of how to combine them is somewhat arbitrary. For the analysis reported here, we developed two methods which agree to better than $1 \%$ on average during solar quiet time. To obtain the results reported here, we used dose data from D2 over its full non-saturating range, and added the dose measured by D1 for the portion of the LET spectrum above $88 \mathrm{keV} / \mu \mathrm{m}$ in $\mathrm{Si}$. (The alternate method takes the dose from D1 over its usable range and adds in the missing low-LET contributions to dose using the D2 data.) There is no D1 $\times$ D2 coincidence requirement in the analysis. The calculation is performed per day and the dose rates are corrected for times when the instrument was powered off, was running its calibration pulser (these data are completely excluded), or during which a SEP event was in progress. Because the LRO orbit is elliptical, and has varied over time, we must also account for the fact that the doses measured by D1 and D2 are not simply from particles entering CRaTER from the zenith direction - particles that come from the nadir-facing side of the instrument can sometimes also reach the detector, when LRO is at or near aposelene and the lunar disk does not fully occupy the instrument's rear-facing field of view. The CRaTER science team provides an hourly-averaged correction factor that is used here to adjust the dose rate to correspond to irradiation in a $2 \pi$ geometry.

There is a small contribution to the measured dose rates from "albedo" particles traveling upward from the lunar surface.
These particles are created when high-energy GCRs interact in the lunar soil and secondaries are produced at large angles. The two layers of TEP in the CRaTER telescope effectively impose a minimum energy requirement on such upward-traveling ions, in that they must traverse $9 \mathrm{~g} / \mathrm{cm}^{2}$ of TEP to reach D2 and deposit energy. For protons and ${ }^{4} \mathrm{He}$ nuclei, the kinetic energy corresponding to this range is about $110 \mathrm{MeV} / \mathrm{nuc}$, which excludes the large majority of albedo particles. Further analysis of the role of albedo particles in the dose rates measured by CRaTER can be found in Spence et al. (2013).

\subsection{The MSL-RAD instrument}

The Radiation Assessment Detector for the Mars Science Laboratory (MSL-RAD) is part of the science payload aboard the Curiosity rover, which has been operating on Mars since August 2012. The instrument is mounted flush with the top deck of the rover. The design and calibration of the RAD instrument were described by Hassler et al. (2012) and Zeitlin et al. (2016), respectively. Results of the RAD observations obtained during the transit to Mars have been reported by Zeitlin et al. (2013), Köhler et al. (2015) and Ehresmann et al. (2016) and several measurements made on the surface of Mars have also been reported (Ehresmann et al., 2014, 2018; Hassler et al., 2014, 2018; Wimmer-Schweingruber et al., 2015; Zeitlin et al., 2018). In the following, we use data from the charged-particle detector $\mathrm{B}\left(\mathrm{MSL}-\mathrm{RAD}_{\mathrm{B}}\right)$.

\subsubsection{MSL-RAD: data products}

In the following we make use of the dosimetry data recorded by the $\mathrm{B}$ detector in the MSL-RAD stack. The A, B, and $\mathrm{C}$ detectors are all segmented silicon diodes of $300 \mu \mathrm{m}$ thickness. For the B diode, only the innermost, central segment is connected; the segment is hexagonal in outline with an area of $1.92 \mathrm{~cm}^{2}$. For purposes of dosimetry, a simple trigger scheme is defined in which all hits in B above the $30 \mathrm{keV}$ energy deposition threshold are recorded. The B detector is read out by electronics with a very wide dynamic range, covering from $20 \mathrm{keV}$ to about $600 \mathrm{MeV}$. A minimum-ionizing charge-1 particle (often referred to as a MIP) will deposit about $120 \mathrm{keV}$ on average, with a most probable deposition of about $80 \mathrm{keV}$. Calibration constants are applied in real time on board, and a running total of deposited energy is kept in memory and updated whenever the $\mathrm{B}$ dosimetry trigger fires. MSL-RAD consumes $4 \mathrm{~W}$ of power while acquiring data, and operates on a variable-length wake/sleep cycle in order to preserve the option for ultra-low average power consumption, as is occasionally required for rover operations (e.g., running at $25 \%$ duty cycle reduces RAD's average power consumption to $1 \mathrm{~W}$ ). Data analysis software running on board divides the duration of the wake cycle into 16 equal intervals, and stores the running total of energy deposits once per interval. For the large majority of Curiosity's surface mission, RAD has operated on a 16-min wake/30-s sleep cycle, thereby storing the dosimetry data once per minute. The data acquired in an observing period are stored locally in non-volatile RAM, and are periodically telemetered to Earth for analysis. In the case of the dosimetry data, the analysis is extremely simple: the energy deposition total for a given measurement (per-minute or per-observation) is converted to the appropriate unit (Joules), divided by the lifetime of the

Page 3 of 20 
Table 1. Relevant data coverage by the instruments for this study.

\begin{tabular}{lcr}
\hline Instrument & Location & Data coverage \\
\hline DOSTEL-1 & ISS & $07.2009-05.2010 \& 05.2012-07.2019$ \\
DOSTEL-2 & Columbus Laboratory & $07.2009-06.2011 \& 05.2012-07.2019$ \\
CRaTER & Moon orbit & $09.2009-07.2019$ \\
MSL-RAD & Cruise phase to Mars & $12.2011-07.2012$ \\
& Mars surface & $08.2012-07.2019$ \\
\hline
\end{tabular}

observation, and finally divided by the mass of $\mathrm{B}$ to yield a dose rate. Because of the small size and mass of $\mathrm{B}$, dose-rate data in short measurement intervals are statistically noisy, and it is generally helpful to average over longer periods (hour to days).

Two other parts of the analysis merit discussion, both of which are related to removal of background. First, RAD detects a steady background of neutrons, gamma-rays, and their interaction products (such as electrons) emanating from Curiosity's radioisotope thermoelectric generator (RTG). The methodology for estimating and removing the RTG contribution to the B detector dose rate has been described in Zeitlin et al. (2016). We estimated that the RTG background produced a rate of $84 \mu \mathrm{Gy} /$ day in MSL-RAD ${ }_{\mathrm{B}}$ during the cruise phase; on the surface, due to different shielding conditions (namely, the absence of the Descent Vehicle directly above RAD), we estimate the contribution to be $67 \mu \mathrm{Gy} /$ day. Second, the Curiosity science payload includes the Dynamic Albedo of Neutrons (DAN) experiment (Mitrofanov et al., 2012) which includes a pulsed neutron generator (PNG) that is fired with some regularity. When fired, the PNG isotropically emits an intense flux of $14 \mathrm{MeV}$ neutrons, some of which go into the Martian soil and produce a flux of moderated, backscattered neutrons that are detected by the two DAN neutron detectors. (The experiment is designed to detect the local presence of hydrogen and other elements such as $\mathrm{Cl}$ and $\mathrm{Fe}$ which have large neutron cross sections.) In the early part of the surface mission, PNG firings caused large excursions in the observed dose rates in $\mathrm{RAD}$ (particularly in the $\mathrm{E}$ detector, which is more sensitive to neutrons than the $\mathrm{B}$ detector), with $\mathrm{E}$ detector dose rates rising by factors of 4-5 above the background GCR rate while the PNG fired. This has markedly declined over the course of the surface mission, with the present increases only factors of 1.2-1.3 above the (now-larger) GCR dose rate in E. Occasionally, the PNG can fire without producing a noticeable increase in RAD dose rates. Our criterion for removing data is simple: (1) if the E detector dose rate is $5 \%$ or more than the $\mathrm{E}$ dose rate in the previous observation, and (2) rover log files show the PNG was firing during all or part of the observation with the enhanced dose rate, the data from that observation are removed from the analysis.

\subsection{Data coverage by the instruments in the years 2009-2019}

The following Table 1 provides for all the instruments under study their relevant data coverage. While data from onboard the ISS (DOSTEL-2 instrument) is provided from 07.2009-06.2011 and subsequent from 05.2012 up to the end of our investigations CRaTER data covers the timeframe from 09.2009-07.2019.
For MSL-RAD we rely on data already measured during the cruise phase to Mars (12.2011-07.2012) and the subsequent data set from the surface of Mars (08.2012-07.2019).

\section{GEANT-4 simulations setup}

The measurements of the dose rates in ISS orbit and free space have been complemented with model calculations performed with the GEANT4 Monte-Carlo framework (Agostinelli et al., 2003; Allison et al., 2006, 2016). Benchmarking model calculations with measurements is essential to establish the trustworthiness of the model results. If the agreement between the model calculations and the experimental outcome is satisfactory, one can also draw additional conclusions from the model results such as energy spectra of individual particle types, relative contributions, etc. This information can be used to derive other relevant quantities that are experimentally not accessible, such as the effective dose equivalent or human organ doses. For the comparison with the DOSTEL data, we calculate the dose rate in a thin silicon detector $(300 \mu \mathrm{m})$ located in a spherical shell, the shielding of which is derived from the shielding distribution of the ISS Columbus module. The cumulative shielding distribution for Columbus is derived from a CAD model of the module and applying a ray-tracing technique for the position of the DOSTEL instrument within COLUMBUS. A more detailed description of the geometry is given in Berger et al. (2018).

Primary GCR spectra were described by the DLR GCR model (Matthiä et al., 2013) for March 2015 to calculate solar maximum like conditions and for January 2019 to calculate solar minimum like conditions. The DLR GCR model is an engineering model derived from the ISO (2004) model providing energy spectra of primary GCR nuclei from hydrogen to nickel. The solar modulation of the GCR intensity is thereby described by a single parameter that can be derived for instance from neutron monitor count rates. A comprehensive validation and comparison with other GCR models has been performed by Norbury et al. (2018) and Whitman et al. (2019).

March 2015 was selected for solar maximum conditions based on $\mathrm{D}_{3} \mathrm{D}_{\mathrm{D}-1 / \mathrm{D}-2}$ measurements and Oulu neutron monitor count rates (http://cosmicrays.oulu.fi/), the latter showing the absolute minimum in monthly average count rates in March 2015. It should be noted that different measurements that reflect the GCR intensity can reach the minimum at different times, depending not only on the detector type and the measured quantity but also on the magnetic and mass shielding conditions under which the detector is measuring. The maximum in solar activity if measured by the sun spot number had already been 
reached in 2014 (see Fig. 2b). January 2019 was selected for solar minimum conditions (intensity maximum) as it showed the maximum values in monthly dose rate as measured by

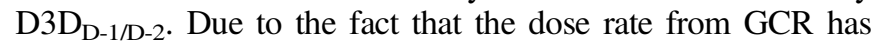
increased only minimally (by less than $2 \%$ ) in the following months (see Fig. 6a) it has been decided not to repeat the calculations for latter times as the difference would be minimal.

For the interplanetary space scenario, the shielding geometry was isotropically irradiated with the full GCR spectrum for primary ions from $\mathrm{H}$ to $\mathrm{Ni}$. The simulated energy range was $10 \mathrm{MeV} / \mathrm{n}-200 \mathrm{GeV} / \mathrm{n}$. The transport of the GCR primaries through the shielding was performed with GEANT4, version 10.03.p03, using a pre-defined reference physics list including all relevant physical processes (namely the QGSP_INCLXX_ HP_EMZ physics list, see: Geant4-Collaboration, 2016; and the relevant GEANT4 website, https://geant4.web.cern.ch/ node/155, for details).

The resulting spectra of primary and secondary particles on the inside of the spherical shielding were registered and converted to dose rates using pre-calculated fluence to dose conversion coefficients for a $300 \mu \mathrm{m}$ silicon detector.

For the calculation of the radiation exposure in the ISS orbit, the identical primary particle spectra have been modified to account for the shielding provided by the magnetosphere and the solid Earth. The latter has been considered by introducing a factor of $f_{\Omega}=0.67$ which corresponds to the solid angle fraction of sky that is not blocked by the planet at an altitude of $400 \mathrm{~km}$ above sea level for a spherical Earth with radius $6371 \mathrm{~km}$. The magnetospheric shielding was estimated by calculating the effective vertical cut-off rigidity $R_{C}$ for each point of the ISS orbit. The cut-off rigidity was calculated using the GEANT4/PLANETOCOSMICS tool for the International Geomagnetic Reference Field 12 (IGRF-12) (Thébault et al., 2015) and the Tsyganenko (1989) model. The combined average shielding can be expressed by the geomagnetic transmission function $f(R)$ (Eq. (1)) for the average orbit which describes the fraction of the primary GCR reaching the orbit in dependence of the rigidity $R$ of a particle, where $H$ is the Heaviside function which is 0 if the argument is negative and 1 otherwise. The sum over all points of the orbit and $R_{C, i}$ is the effective vertical cut-off rigidity at each point. Here, a 30 day orbit generated with SPENVIS (https://www.spenvis.oma.be) with an inclination of $51.6^{\circ}$ and a one minute resolution was used, i.e. $N=30 \times$ $24 \times 60=4.32 \times 10^{4}$,

$$
f(R)=f_{\Omega} \sum_{i=0}^{N} H\left(R-R_{c, i}\right) / N
$$

The resulting transmission function $f(R)$ is shown in Figure 1.

Folding the primary GCR spectra in free space with $f(R)$ yields the primary GCR spectra averaged over the ISS orbit. These are the spectra incident on the outside of the ISS. The spectra were then transported using the above described simulation setup to calculate the primary and secondary particle spectra inside the shielding, and to derive the resulting dose rates to be expected in the Columbus laboratory of the ISS. The results of the calculations for both interplanetary space and ISS orbit for solar minimum and maximum conditions are provided in Section 4.6.

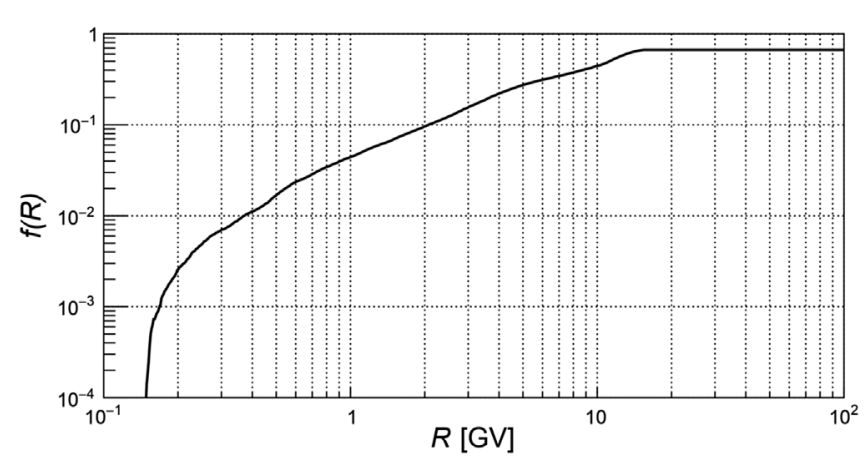

Fig. 1. Transmission function $f(R)$ for the average ISS orbit including geomagnetic shielding and a shadowing factor $f_{\Omega}=0.67$ for an altitude of $400 \mathrm{~km}$.

\section{Results}

In the following we will provide at first results from measurements on board the ISS for the total daily dose values and describe procedures for the separation of the GCR and SAA contributions. This will be followed by data plots generated using various cuts for $L$-values (McIlwain, 1961) and the idea of using high $L$-value cuts for ISS orbits as surrogate for a free space analogue. The hereby applied $L$ parameter has been introduced by McIlwain (1961) to order measurements in a geomagnetic field by defining a quantity that is identical and constant for trapped particles on a given shell. For a dipole field the $L$ parameter is equal to the distance from the Earth center in units of the Earth radius if one follows the magnetic field line at the point of interest to the equator. In addition we will discuss SPE data identified by applying $L$-value cuts on board the ISS and compare them to data measured with other instruments in LEO, the Moon and on the surface of Mars. The final part of this section will be devoted to the comparison of GCR data for ISS, the Moon, the cruise phase to Mars and the surface of Mars for the relevant time periods of data provided by the instruments (as given in Table 1).

\subsection{The DOSTEL instruments - baseline data}

The baseline data for the ISS are the data measured with the DOSTEL-1 and the DOSTEL-2 instruments in the frame of the DOSIS (2009-2011) and the DOSIS 3D (2012 -) projects. The relevant data are presented in Figure 2.

Figure 2 provides in Figure $2 \mathrm{a}$ the altitude of the ISS from 2009 to 2019 in Figure 2b the monthly (blue) and smoothed (red) sunspot numbers and in Figure 2c the Oulu NM count rate in counts/s. In addition data is shown in Figure $2 \mathrm{~d}$ for the daily dose rate measured with the DOSTEL-1 instrument and in Figure $2 \mathrm{e}$ for the daily dose rate measured with the DOSTEL-2 instruments for the timeframe under study.

\subsection{GCR/SAA separation}

As the ISS travels around the world it sees the variation of the GCR due to the Earth's magnetic shielding, which is the baseline background radiation we measure on board the ISS. 

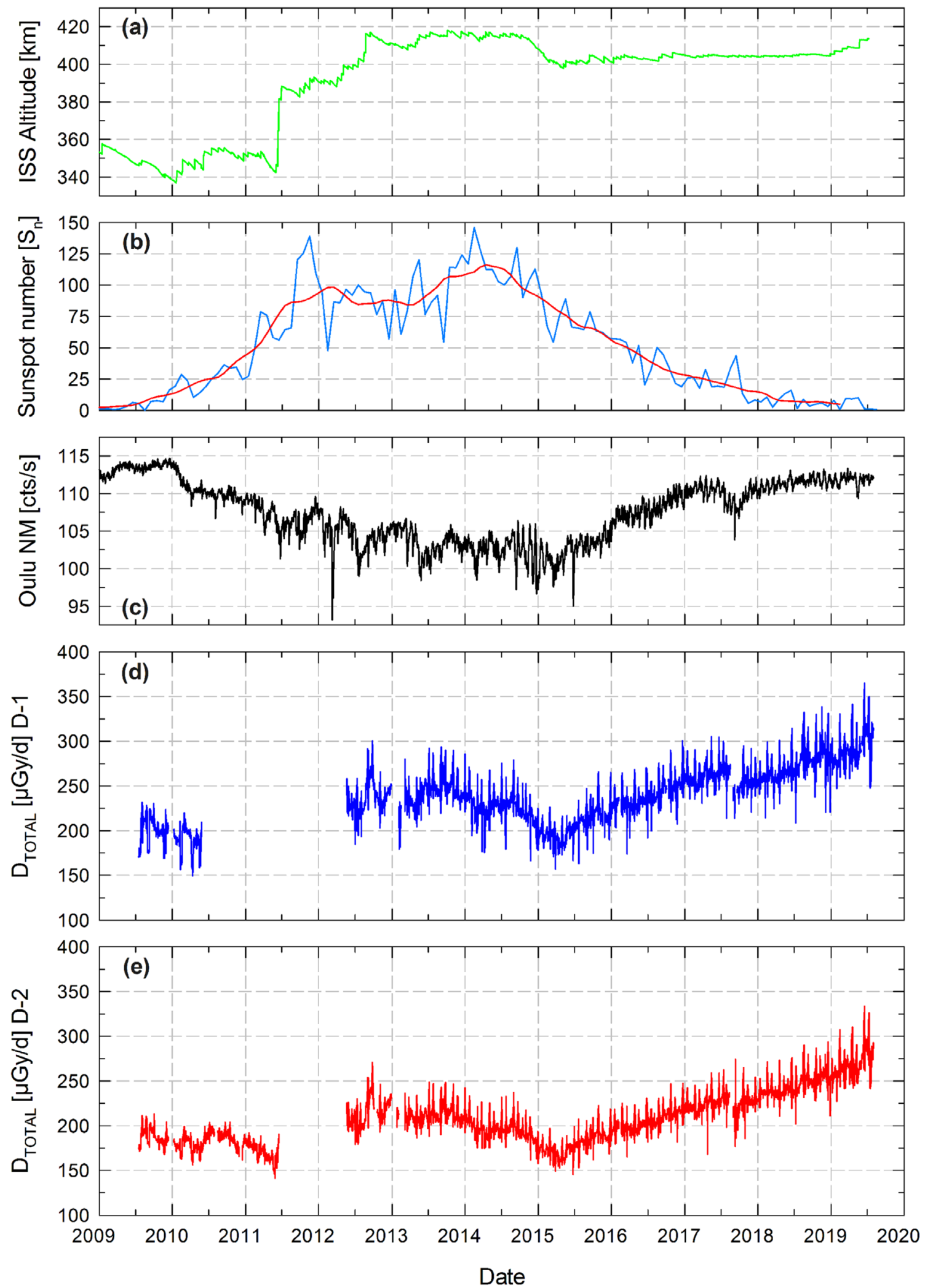

Fig. 2. (a) The altitude of the International Space Station from 2009 to 2019; (b) monthly (blue) and smoothed (red) sunspot numbers (data from: http://www.sidc.be/silso/datafiles); (c) Oulu NM count rate in counts/sec (data from: http://cosmicrays.oulu.fi/); (d) DOSTEL-1 (D-1) total absorbed daily dose rate; and (e) DOSTEL-2 (D-2) total absorbed daily dose rate. 


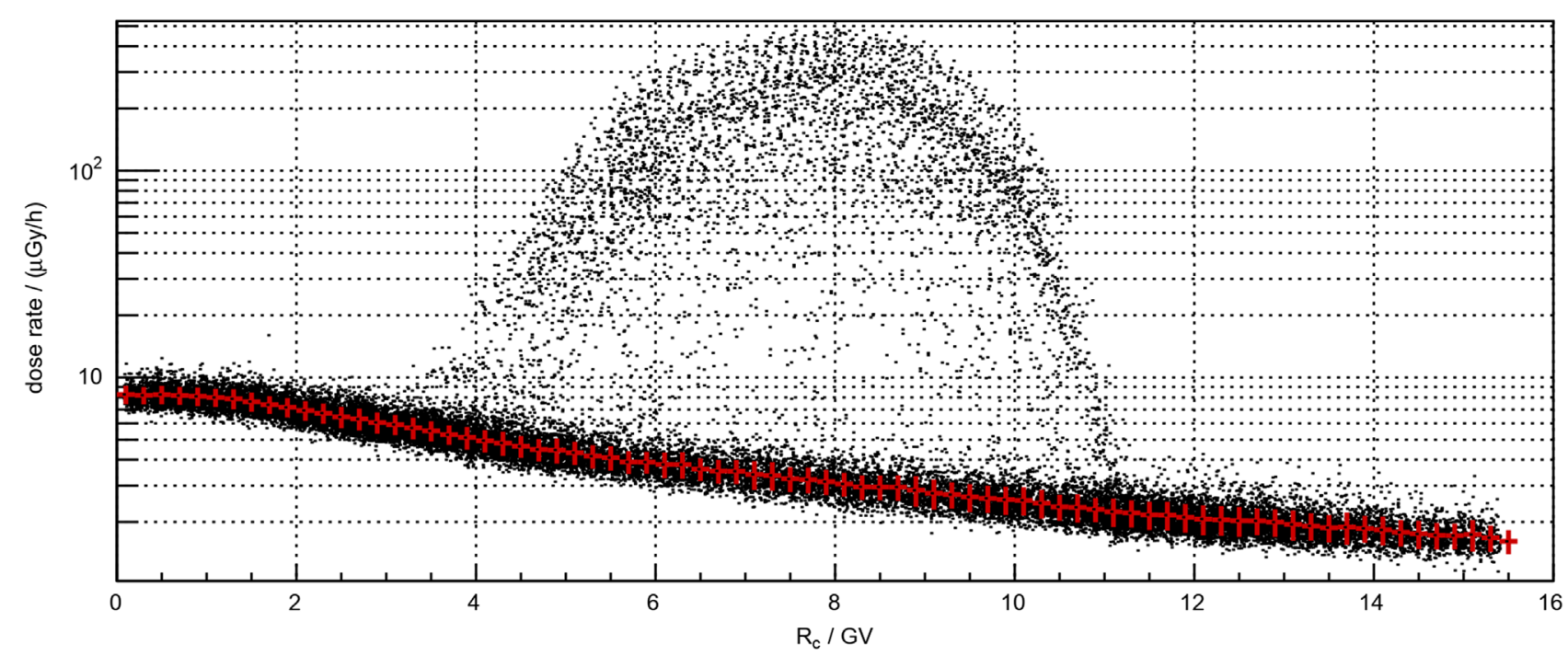

Fig. 3. DLR GCR/SAA dose rate for $\mathrm{D} \mathrm{D}_{\mathrm{D}-1}$ instrument for March 2015 ; dose rate versus $R_{C}$ with the mean and standard deviation of the SAA-free subset given in red.

In addition the ISS passes through the SAA on average eight times a day, with half of the passes being ascending ("southnorth") and the other half descending ("north-south") parts of the orbit. While passing through the SAA, the count and dose rate increase drastically while at the same time the GCR contributions remain as the background. Up to now there is no common procedure used by relevant instruments measuring on board the ISS to clearly separate the GCR from the SAA contributions. In the following we will describe two procedures developed by DLR and NASA-SRAG (Space Radiation Analysis Group) for the separation of the GCR and the SAA environment and shortly discuss their features.

\subsubsection{DLR: GCR/SAA separation}

The separation of the contributions of GCR and radiation belt particles in the SAA is performed on the basis of the cut-off rigidity $\left(R_{C}\right)$ dependence of the dose rates. The method that is presented in the following is applicable to arbitrary time intervals for which the statistical uncertainty of the measurement is sufficiently small provided that the GCR intensity is approximately constant. Below, the results for one month intervals will be presented.

For each time interval, the following steps were applied:

1. Apply a loose geographical cut to select measurements outside the SAA and to get a subset without SAA contributions; in this analysis data points recorded south of $10^{\circ} \mathrm{N}$ latitude and at longitudes between $90^{\circ} \mathrm{W}$ and $25^{\circ} \mathrm{E}$ are excluded (cf. Fig. 4a).

2. The geographical coordinates of each measurement are converted to the corresponding cut-off rigidity $R_{C}$ and the measurements are grouped into $R_{C}$ intervals (Fig. 3) here we use intervals of $0.2 \mathrm{GV}$.

3. For each interval, the mean and the standard deviation of the dose rate are calculated.
4. Each measurement of the full data set is categorized as inside or outside the SAA based on the deviation of the measurement from the mean of the cut-off rigidity interval; here we apply a cut of 4 standard deviations from the mean.

5. For each measurement that is categorized as inside the SAA, the mean of the corresponding cut-off rigidity interval of the SAA free subset is subtracted and added to the set of GCR data with the corresponding timestamp and coordinates as illustrated in Figures $4 \mathrm{~b}$ and $4 \mathrm{c}$.

Figure 3 illustrates the cut-off rigidity dependence of the dose rate exemplarily for March 2015 for the $\mathrm{D}^{2} \mathrm{D}_{\mathrm{D}-1}$ instrument data. The individual measurements are indicated by the black markers and the red line shows the mean and the standard deviation in each $0.2 \mathrm{GV}$ cut-off rigidity interval for the reduced data set applying the geographical selection.

Figure 4 shows exemplary results for $\mathrm{D}^{2} \mathrm{D}_{\mathrm{D}-1}$ instrument and March 2015 applying the four standard deviations selection criterion on the data. From top to bottom are: Figure 4a the full data set including all measurements; the cut-off rigidity is illustrated by the contour lines and the geographical cut that is applied to create the initial data set for the cut-off rigidity dependence of the GCR dose rate is illustrated by the red shaded box; Figure $4 \mathrm{~b}$ all data points categorized as GCR with the measurements from within the SAA removed and replaced by the mean value of the dose rate from the corresponding cut-off rigidity interval (as given in Fig. 3) (data is given as $\mathrm{GCR}_{\mathrm{DLR}}$ in Table 2); Figure $4 \mathrm{c}$ measurements categorized as SAA with the mean dose rate in the corresponding cut-off rigidity bin subtracted (data is given as $\mathrm{SAA}_{\mathrm{DLR}}$ in Table 2).

\subsubsection{NASA: GCR/SAA separation}

We use a model-based definition, thereby preventing any sensitivity to localized shielding inside or outside the vehicle 

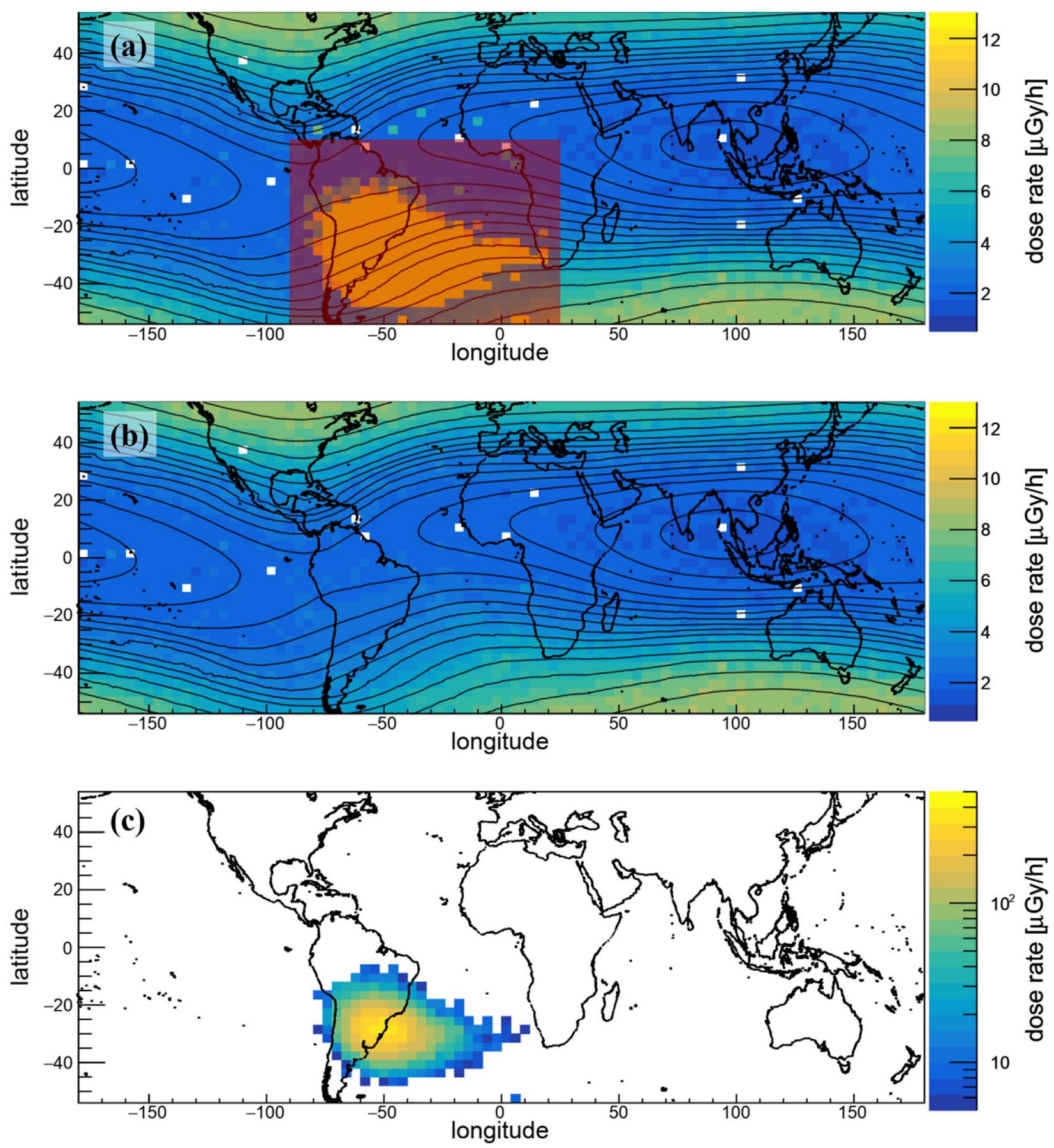

Fig. 4. $D 3 D_{D-1}$ dose rate measurements and SAA separation, exemplarily for March 2015: (a) full data set, geographical cut illustrated by the

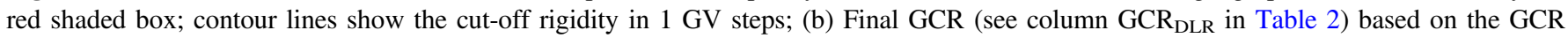
contribution outside the SAA $\left(\mathrm{GCR}_{\mathrm{w} / \mathrm{o}}\right.$ - Table 2) plus the GCR contribution inside the SAA $\left(\mathrm{GCR}_{\mathrm{in} \mathrm{SAA}}\right.$ - Table 2); and (c) SAA (see column $\mathrm{SAA}_{\mathrm{DLR}}$ in Table 2$)$ based on the SAA contribution with GCR $\left(\mathrm{SAA}_{\mathrm{w}}-\right.$ Table 2$)$ minus the GCR contribution inside the $\mathrm{SAA}_{(\mathrm{GCR}}$ in SAA Table 2).

that utilizes the geomagnetic field intensity $(B)$ and Mcllwain (McIlwain, 1961) $L$-shell parameter $(L)$ as attributed to ISS's trajectory. We have categorized the SAA using $|B|<23 \mu \mathrm{T}$ and $L<3$; (see also Berger et al., 2018) the remaining environment is thereby categorized as GCR. The ISS trajectory has been calculated using the instruments' timestamps and SGP4 (Simplified General Perturbation model); for improved accuracy, we calculate the geomagnetic field using IGRF-12 (Thébault et al., 2015) and $L$-shell at each point in the trajectory. Figure 5 provides the relevant data. 
(a)

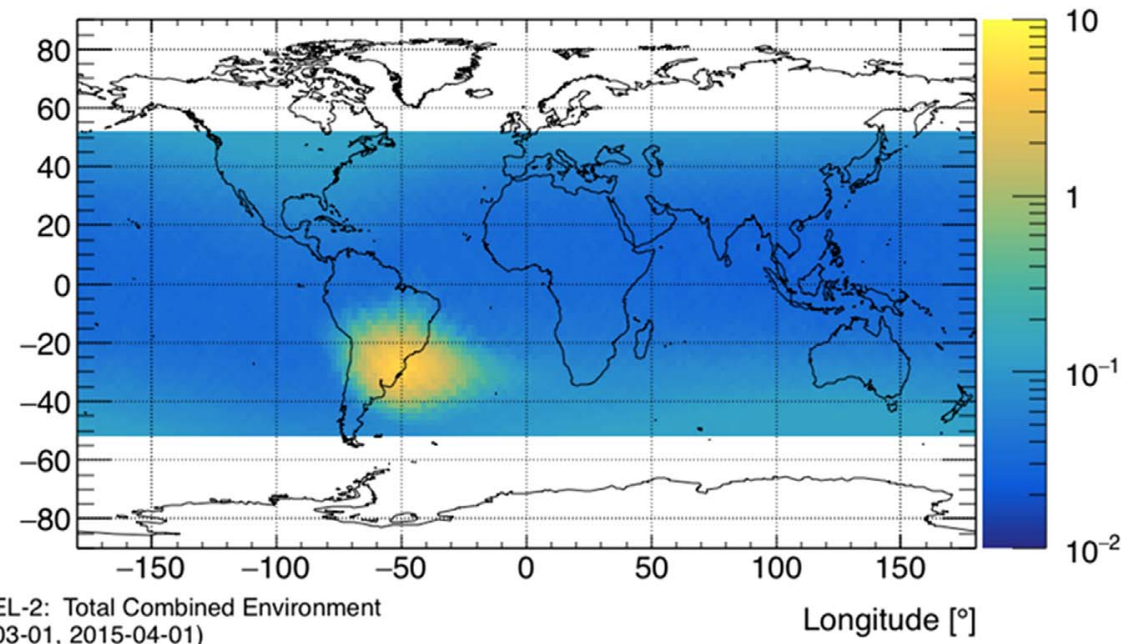

$$
\text { 胥 }
$$

DOSTEL-2: Total Combined Environment [2015-03-01, 2015-04-01)

일

(b)

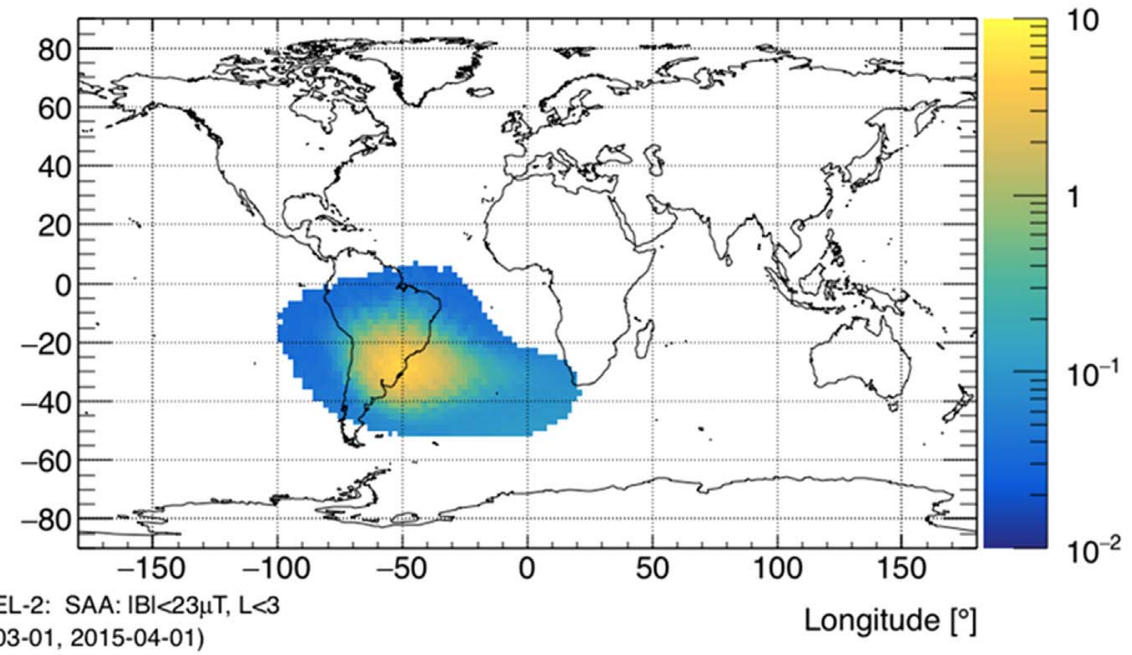

[2015-03-01, 2015-04-01)

일

(c)

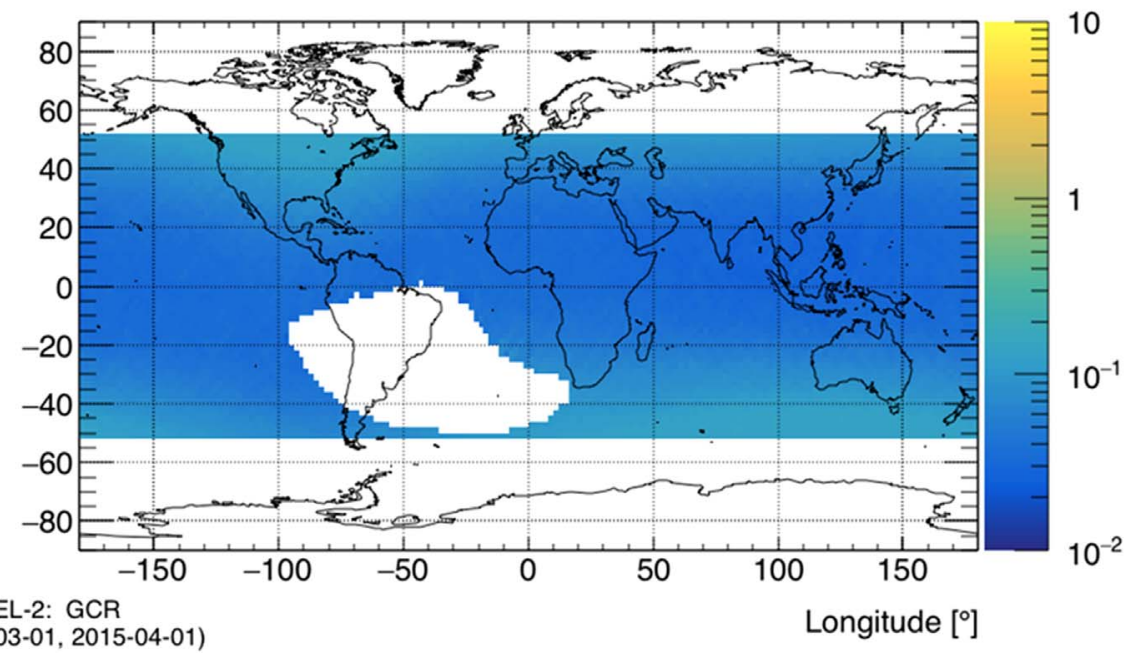

DOSTEL-2: GCR
[2015-03-01, 2015-04-01)

Fig. 5. Example data for the $\mathrm{D} \mathrm{D}_{\mathrm{D}-2}$ instrument showing the dose rate over the orbit for March 2015 (a) Full; (b) SAA (see Table 2 column SAA $_{\text {NASA }}$ ) cut; and (c) GCR (see Table 2 column GCR $_{\text {NASA }}$ ).

\subsubsection{Comparison of the two procedures}

Table 2 provides a comparison of the two procedures for two six months' time periods at the beginning of 2015 and
2019. Shown are the daily average dose values derived by DLR for the GCR dose without contribution from the SAA crossings $\left(\mathrm{GCR}_{\mathrm{w} / \mathrm{o}}\right)$, the respective SAA dose with the contribution from GCR $\left(\mathrm{SAA}_{\mathrm{w}}\right)$ the average GCR dose inside the SAA 
Table 2. Comparison of GCR and SAA doses derived by DLR and NASA-SRAG procedure for two six months' time periods in 2015 and 2019.

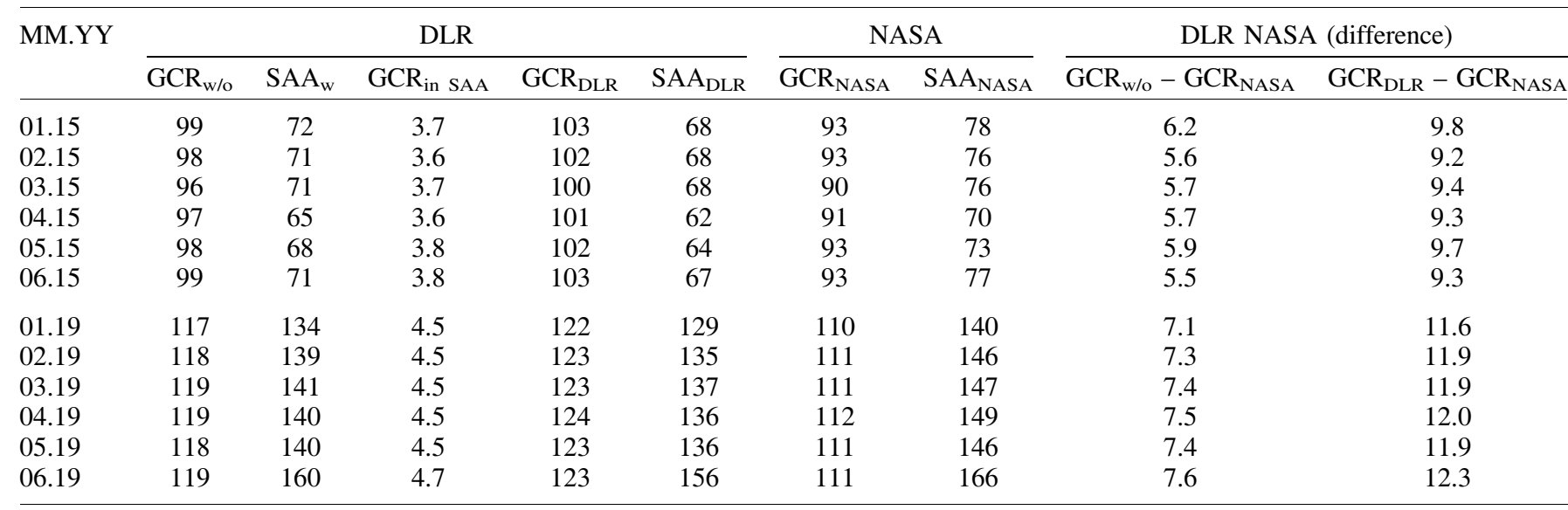

Note: All dose values in $\mu$ Gy/day.
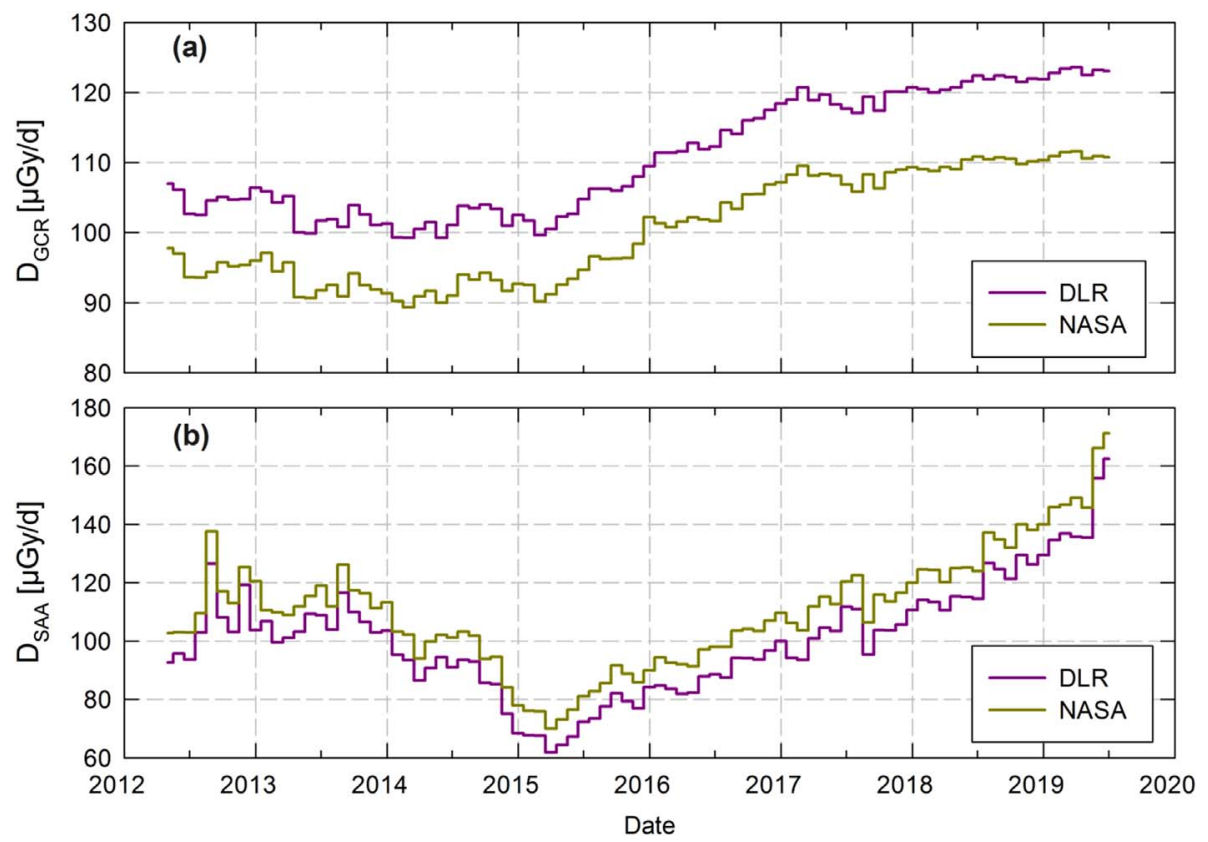

Fig. 6. (a) Daily GCR dose for DLR (dark pink) and NASA (dark yellow) procedure and (b) daily SAA dose for DLR (dark pink) and NASA (dark yellow) procedure.

$\left(\mathrm{GCR}_{\text {in SAA }}\right)$ and the respective full daily GCR dose $\left(\mathrm{GCR}_{\mathrm{DLR}}\right)$ and SAA dose $\left(\mathrm{SAA}_{\mathrm{DLR}}\right)$. The NASA-SRAG data shows the daily GCR $\left(\mathrm{GCR}_{\mathrm{NASA}}\right)$ and SAA dose $\left(\mathrm{SAA}_{\mathrm{NASA}}\right)$ based on the respective $|B|$ and $L$ value cut.

If one compares the difference in GCR dose values between the DLR $\mathrm{GCR}_{\mathrm{w} / \mathrm{o}}$ and the NASA $\mathrm{GCR}_{\mathrm{NASA}}$ it is shown, that the additional GCR dose attributed by NASA to the SAA (6.2-7.6 $\mu \mathrm{Gy} /$ day) is due to the more conservative procedure NASA-SRAG performs. This is easy visible by comparing the area of the SAA shown in Figure 4c for DLR and in Figure $5 \mathrm{~b}$ for NASA-SRAG. If one compares the final DLR GCR doses $\left(\mathrm{GCR}_{\mathrm{DLR}}\right)$ with the NASA-SRAG $\left(\mathrm{GCR}_{\mathrm{NASA}}\right)$ the difference is in average $9.6 \mu \mathrm{Gy} /$ day during solar maximum and $11.9 \mu \mathrm{Gy} /$ day during solar minimum conditions, whereas the additional GCR dose within the SAA $\left(\mathrm{GCR}_{\text {in }} \mathrm{SAA}\right)$ contributes the added around $4 \mu \mathrm{Gy} / \mathrm{day}$.

The long term comparison of the GCR and SAA dose values derived by the two procedures and shown in Table 2 for the two six months' time periods is provided in Figure 6. Figure 6a shows the difference in GCR dose values while Figure $6 \mathrm{~b}$ gives the data for the SAA contribution. As mentioned above, the baseline data set for the current DLR procedure requires about one month of data, therefore for short term (daily comparisons), the DLR procedure is currently not applied. Its advantage is that it provides an instrument specific GCR dose where the falsely to the SAA attributed GCR contributions are calculated and added to the GCR dose. For comparison with other instruments on board the ISS the NASA-SRAG $|B| / L$ cut is straightforward and the real 

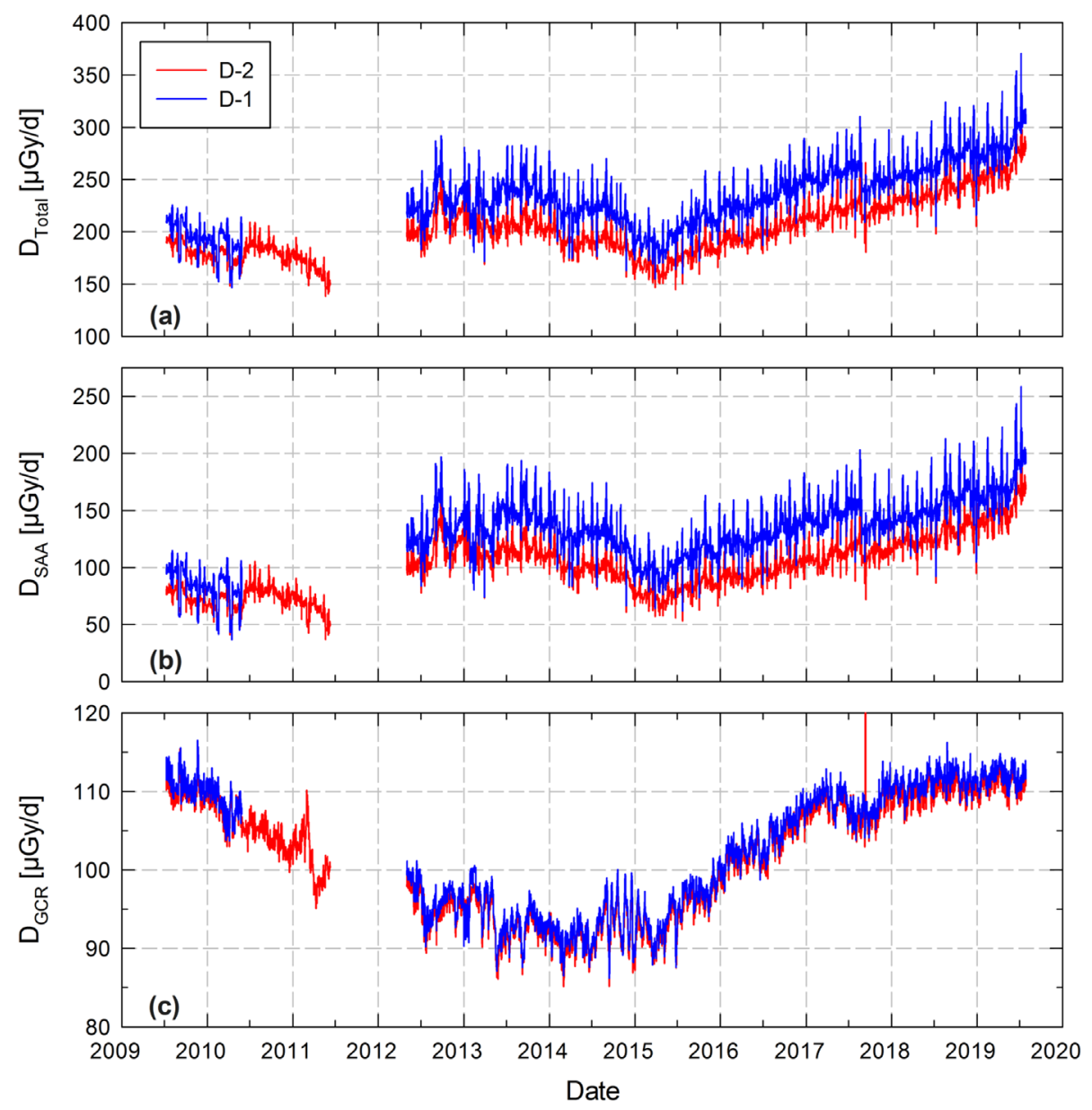

Fig. 7. (a) Total daily dose measured with D-1 (blue) and D-2 (red); (b) SAA contribution to the daily dose; and (c) GCR contribution to the daily dose.

GCR dose is only underestimated by $\sim 9 \%$. Also the NASASRAG procedure is purely based on the outside environment, therefore making it easier to compare instruments inside the ISS which are positioned behind various shielding. So while both procedures have their advantages and disadvantages, the NASA-SRAG procedure is independent from measurement data and only uses the $|B| / L$ separation procedure, while the DLR procedure uses the measurement data as baseline. Comparison of data measured with the DOSTEL instruments and the ISS-RAD instruments on board the ISS based on the NASA-SRAG procedure has already been successfully performed for the SPE in September 2017 (see Berger et al., 2018). In the following parts of the manuscript the daily dose values and the respective $L$-value cuts are based on the NASA-SRAG procedure while the comparison of GCR dose with GEANT4 calculation is based on the DLR procedure.

\subsection{Results based on the NASA B/L cut}

The following data evaluation for the DOSTEL data is based on the primary D-1 and D-2 measured data (as given in Fig. 2) further evaluated using the NASA $|B| / L$ cut for GCR/SAA separation. Figure 7 provides for the D-1 and the D-2 instruments the total daily absorbed dose values (Fig. 7a), the daily dose contributions from the SAA crossings (Fig. 7b) and the daily GCR dose values (Fig. 7c) for the time period under study. What can be clearly seen in Figure $7 \mathrm{c}$ is the peak in dose rate in September 2017, which relates to the Solar Particle Event from September 102017 (Berger et al., 2018; Ehresmann et al., 2018; Hassler et al., 2018; Jiggens et al., 2018; Zeitlin et al., 2018) and is attributed as GCR dose due to the $|B| / L$ cut procedures.

The results as provided in Figure 7c for the daily GCR dose measured with the D-2 instrument in the years 2009-2019 will now be used as the baseline data for the further in- depth analysis starting in the next part of this work.

\subsubsection{L-value GCR cuts}

As indicated and shown in Figure 7c we will now further apply an $L$-value cut for the GCR daily dose values. The $L$-value cut enables us to generate plots for various geomagnetic

Table 3. $L$-value cuts and respective $R_{C}$.

\begin{tabular}{lcc}
\hline$L_{\text {REGION }}$ & $L_{\text {VALUE }}$ & $R_{C}(\mathrm{GV})$ \\
\hline$L L$ & $0 \leq L<2$ & $>3.63$ \\
$M L$ & $2 \leq L<4$ & 3.63 to $<0.91$ \\
$H L$ & $4 \leq L$ & $\leq 0.91$ \\
\hline
\end{tabular}



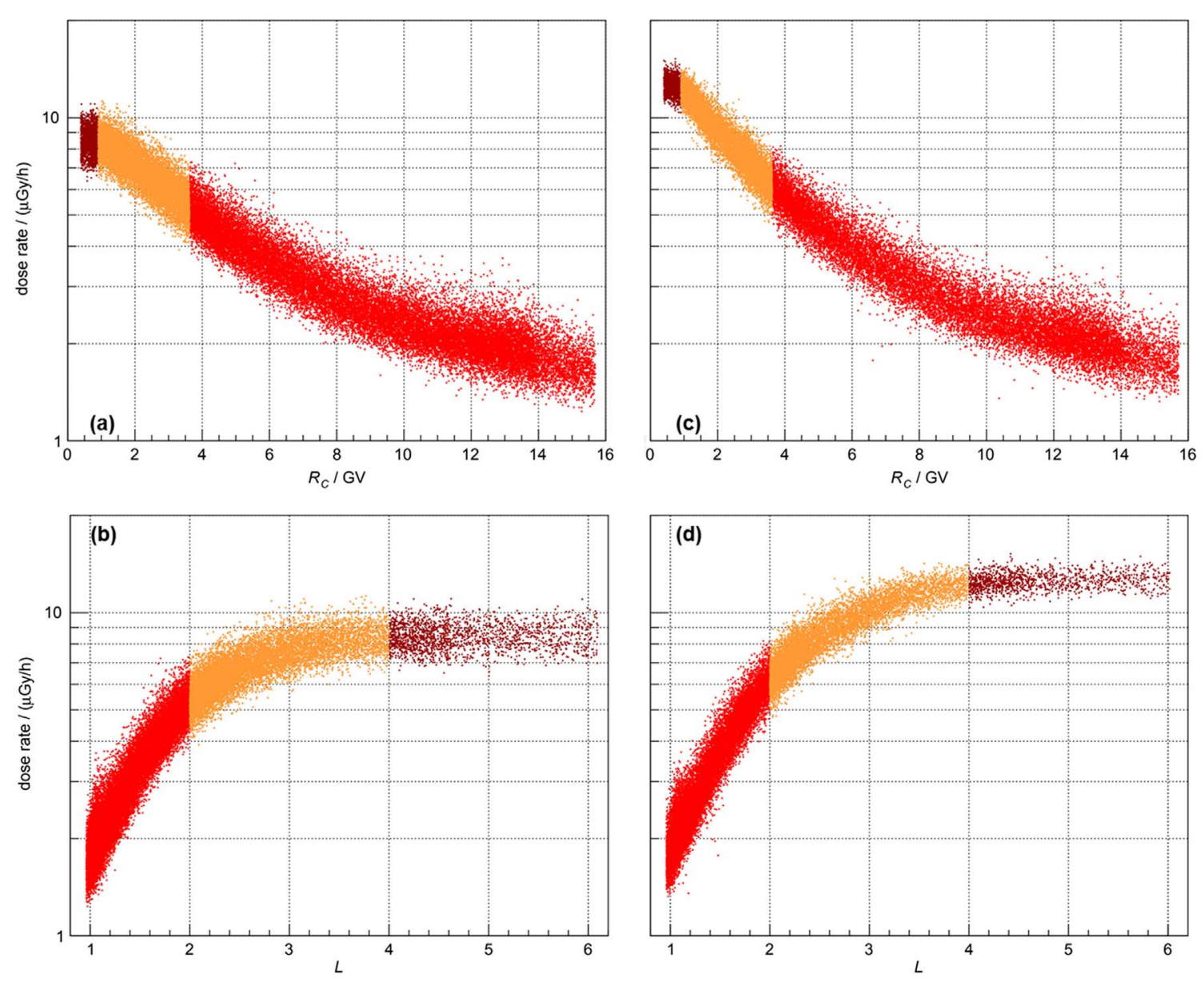

Fig. 8. (a) GCR dose rate versus $R_{C}$ for December 2014; (b) GCR dose rate versus $L$-value for December 2014; (c) GCR dose rate versus $R_{C}$ for January 2019; and (d) GCR dose rate versus $L$-value for January 2019. Note: the respective $L L, M L$ and $H L$ regimes have been given in relevant color coding $\left(L L=\right.$ red, $M L=$ orange and $H L=$ dark red). Color coding for the respective $L$ values is also reflected for the $R_{C}$ data.

shielding conditions. Similar investigations using the $L$-value cut as surrogate and possibility to have a free space analogue have already been presented in Narici et al. (2017) and Zeitlin et al. (2019b), where the high $L$-value dose values from the DOSTEL instruments have been compared to data from the ALTEA instrument (Narici et al., 2017) and on the other hand the ALTEA, CRaTER, and MSL-RAD LET spectra have been compared to each other (Zeitlin et al., 2019b).

For the $L$-value separation, three different regimes were applied as provided in Table 3 denoted as $L L, M L$ and $H L$. The respective vertical cut-off rigidities $R_{C}$ for the three regimes are also listed in Table 3. For the calculations of the $R_{C}$ the relevant approximation of Smart and Shea (2005) with $R_{C}=14.5 / L^{2}$ was applied.

Figure 8 shows the $\mathrm{D} \mathrm{D}_{\mathrm{D}-2}$ dose rate versus $R_{C}$ and versus $L$ for two time intervals, namely in December 2014 (Figs. 8a and 8b) and in January 2019 (Figs. 8c and 8d). ${ }^{1}$ The relevant $R_{C}$ intervals and the respective $L L, M L$ and $H L$ cuts are shown with color codes (red, orange, dark red). Noticeable is the increase in the absorbed dose rate especially for the $M L$ and $H L$ cuts from Figures $8 \mathrm{~b}$ to $8 \mathrm{~d}$ due to the change in the solar

\footnotetext{
${ }^{1}$ Note that these data are shown in units of $\mu \mathrm{Gy} / \mathrm{h}$ rather than $\mu \mathrm{Gy} /$ day as in Figure 7 .
}

cycle - going from solar maximum conditions in Figure $8 \mathrm{~b}$ to solar minimum conditions in Figure 8d. Further on the $H L$ cuts clearly show a plateau region in the absorbed dose rates resulting in average hourly dose rates of $8.5 \pm 0.02 \mu \mathrm{Gy} / \mathrm{h}$ for December 2014 and $12.53 \pm 0.02 \mu \mathrm{Gy} / \mathrm{h}$ for January 2019 .

Figure 9a provides the results from these cuts for the D-2 instrument in the timeframe under study. While the data for the low and medium $L$-value cut are close to each other the high $L$-value cut shows daily dose values of around $10 \mu \mathrm{Gy}$. The reason for this is of course the occupancy of the ISS in the relevant cut intervals. While for the low $L$ cut we have an average occupancy of $880 \mathrm{~min} /$ day this is decreased to $360 \mathrm{~min} /$ day for the medium $L$-value cut and to around $56 \mathrm{~min} /$ day for the high $L$-value cut.

We can now calculate a daily dose value as if the ISS would spend the full day (FD) in the relevant $L$-value regimes. This data is presented in Figure 9b. It clearly shows that the $H L$ cut dominates the daily dose values, while the low $L$-value cut would result in daily dose values of around $75 \mu \mathrm{Gy} / \mathrm{day}$, a factor of 2-3 less than the other $L$-value regimes, with only weak dependence on the state of the solar cycle. The difference in the $L$-value regimes, and thereby also in the geomagnetic shielding of the Earth are better reflected in Figure 9c where the relevant data provided in Figure $9 \mathrm{~b}$ is normalized to the first 

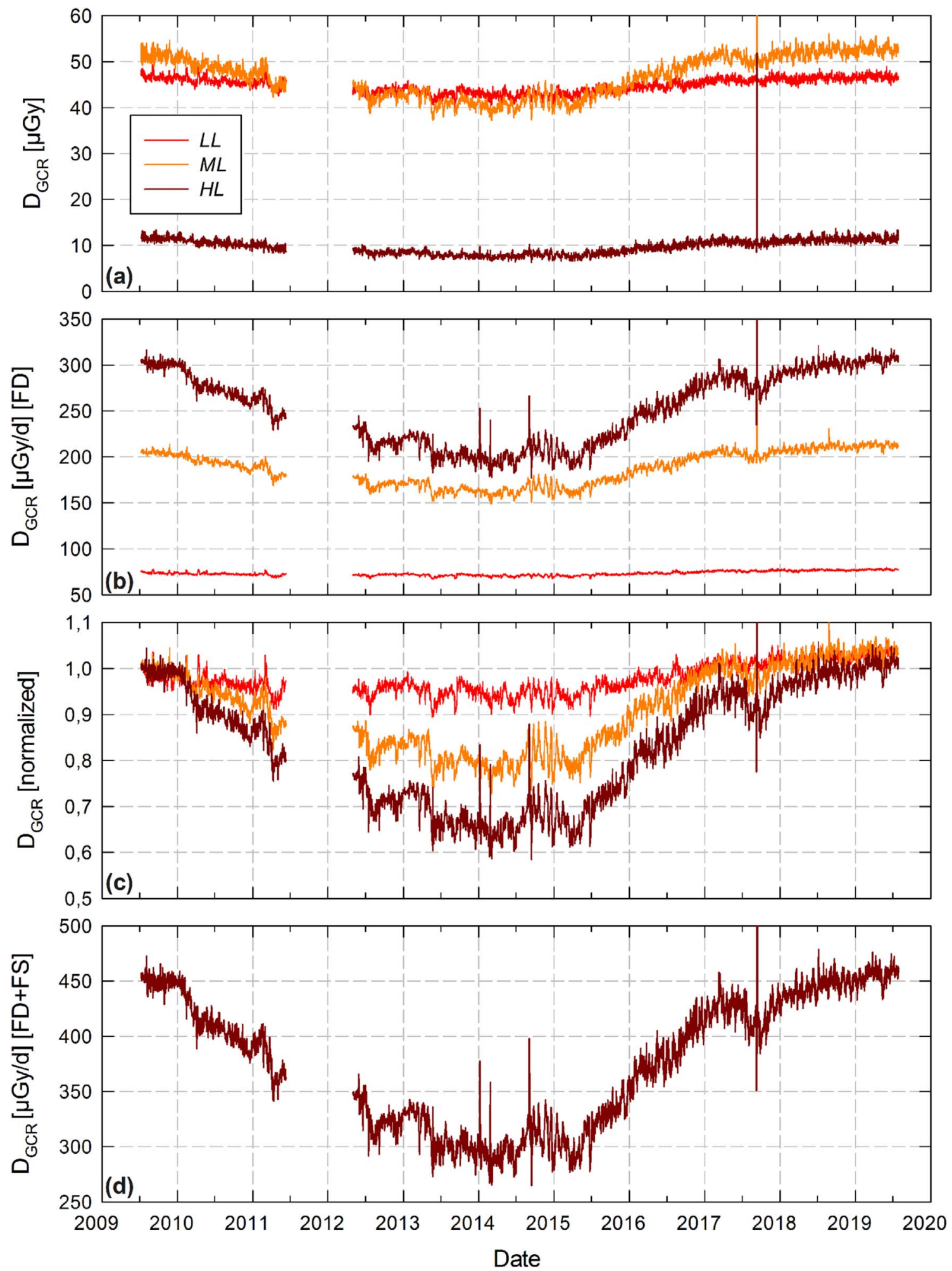

Fig. 9. (a) Daily GCR dose based on low, medium and high $L$-value cut; (b) GCR dose extrapolated to a full day (FD) occupancy of the ISS in the respective $L L, M L$ and $H L$-value regimes; (c) normalized dose values as given in (b); and (d) daily GCR free space (FS) dose for an instrument with the relevant shielding as for the Columbus laboratory. 
data entry. While low $L$-value data only changes up to $10 \%$ during the solar cycle the high $L$-value data is reduced to a value of around 0.6 at solar maximum conditions. In the next step we use the GCR daily data for $L>4$ and project them to free space (FS). For this, the effect of Earth's shadow on the GCR dose has to be accounted for. At an altitude of around $400 \mathrm{~km}$, the ISS sees only around $67 \%$ of the omnidirectional GCR dose from free space. Therefore if this is taken into account one can calculate a free space dose of the detector data, which is given in Figure 9d. The FS dose reflects the dose that would be measured with a DOSTEL instrument for the relevant shielding (inside Columbus) if a spacecraft with this shielding flew in free space.

\subsection{SPE seen in the new evaluated data}

In the years under study (2009-2019) we only saw two Solar Particle Events which were classified as ground level enhancements (GLE), namely GLE 71 on 17 May 2012 and GLE 72 on 10 September 2017. For the relevant list off GLEs the reader is referred to https://gle.oulu.fi/ and Usoskin et al. (2016). These two events have also been seen with radiation detectors inside the ISS. GLE 71 data was for example measured with the ALTEA instrument (Berrilli et al., 2014) and GLE 72 data was documented by the $\mathrm{D} \mathrm{D}_{\mathrm{D}-1 / \mathrm{D}-2}$ and the NASA ISS-RAD ${ }_{B}$ instruments (Berger et al., 2018). Notable to mention is that GLE 72 was also seen by CRaTER (Schwadron et al., 2018) and by MSL-RAD on the surface of Mars (Ehresmann et al., 2018; Hassler et al., 2018; Zeitlin et al., 2018). In addition to these two GLEs further SPEs have been measured in LEO in the years under study with various instruments as for example the AMS-02 detector mounted outside the ISS (Bindi, 2016) and the PAMELA instrument (Bruno et al., 2018) flying on a quasi-polar elliptical orbit.

Taking all these information about SPEs into account we were aiming to use the applied $L$-value cuts on the $\mathrm{D} 3 \mathrm{D}_{\mathrm{D}-1 / \mathrm{D}-2}$ data to search for SPE events within this data set knowing that possible SPE contributions start to appear approximately at $L>3$ (see also Fig. 5 in Berger et al., 2018). The relevant SPE contributions were, of course, already present in the data, but had not previously been discerned. Results of the newly identified SPEs with the DOSTEL instruments inside the ISS are provided in Figure 10 and Table 4. Figure 10 provides in Figure 10a the Oulu NM count rate for January 2013 to January 2015, in Figure 10b the GOES-15 proton flux for energies $>100 \mathrm{MeV}$, in Figure $10 \mathrm{c}$ the daily dose rate measured with the $\mathrm{CRaTER}_{\mathrm{D} 1 / \mathrm{D} 2}$ detectors circling around the Moon, in Figure 10d the daily dose rates for the $H L$-value cuts for the $\mathrm{D} 3 \mathrm{D}_{\mathrm{D}-1 / \mathrm{D}-2}$ instruments for the same time period and in Figure 10e the daily dose rate as measured with the MSL-RAD ${ }_{B}$ instrument on the surface of Mars. Four additional SPEs have been identified within the $\mathrm{D} 3 \mathrm{D}_{\mathrm{D}-1 / \mathrm{D}-2}$ data (also given with event numbers (\#) 1-4 in Fig. 10d and in the event column of Table 4 showing the events from 22 May 2013, 06 January, 25 February and 01 September 2014) which have also been seen with CRaTER in Moon orbit.

Table 4 provides the overview of these four events measured with $\mathrm{D}_{3} \mathrm{D}_{\mathrm{D}-1 / \mathrm{D}-2}$ relating them to the same events measured with AMS-02 (Bindi, 2016), PAMELA (Bruno et al., 2018) and SOHO/EPHIN (Paassilta et al., 2017). Further on the relevant CRaTER (Fig. 10c) and MSL-RAD (Fig. 10e) events for the time period are also provided. While CRaTER observed all four events, MSL-RAD only saw the events in January and September 2014, due to the magnetic Sun-EarthMars connection for these time periods (see also: http:// helioweather.net/archive/2014/index.html). Interesting to note is also that the January 2014 event (see also Thakur et al., 2014) was later quantified by Mishev et al. (2017) as one of the two Sub-GLE events in this solar cycle.

To further illustrate the SPE contributions at high $L$-values Figures $11 \mathrm{a}-11 \mathrm{~d}$ show the dose rate versus $L$-value plots for the four events measured by the $\mathrm{D}_{3} \mathrm{D}_{\mathrm{D}-2}$ instrument. Provided is always the pre-event background dose rate (black) and the event dose rate (red) for the events. The relevant averaged dose rates for $L>4$ for these pre-event and event days are further provided in Table 5. We can see an increase of dose rates for $L>4$ between $25.4-32.3 \%$ for these four events.

These four newly identified events seen inside the ISS with the $\mathrm{D}_{3} \mathrm{D}_{\mathrm{D}-1 / \mathrm{D}-2}$ instruments have been according to Cohen $\&$ Mewaldt (2018) amongst the strongest events in solar cycle 24 in terms of proton fluence $>10 \mathrm{MeV}$. Even though they were not strong enough to cause a GLE on Earth we can state, that, based on the relevant magnetic connection between Sun-Earth and Mars two of these events (the sub-GLE event in January and the September 2014 event) have also been seen on the surface of Mars making them the first events observed simultaneously inside the ISS, the Moon and on the Mars surface.

\subsection{GCR - the full picture}

In the following we will provide, in addition to the dose values for the ISS orbits and the relevant Free Space analogue data, a comparison with CRaTER and MSL-RAD for data coverage as given in Table 1. CRaTER data has been reevaluated for the D1/D2 dose for this endeavor from 2009 to mid of 2019 as discussed in Section 2.2.1.

Figure 12a provides the daily GCR dose for the ISS measured with the $\mathrm{D}_{3} \mathrm{D}_{\mathrm{D}-2}$ instrument, the daily $\mathrm{CRaTER}_{\mathrm{D} 1 / \mathrm{D} 2}$ dose already translated to the surface of the Moon from the start of the CRaTER measurement campaign, and the daily dose measured on the surface of Mars by the MSL-RAD ${ }_{B}$ detector.

We now extrapolate the data given in Figure $12 \mathrm{a}$ to Free Space. For ISS orbit this is based on the $L$-value cuts (as provided in Fig. 9d). For the surface of the Moon and the surface of Mars, this accounts to taking into account half of the field of view due to the surface. It has to be noted that for the case of Mars, this is a first-order approximation, as the radiation environment on the surface is also dependent on changes in the Martian seasonal pressure cycle. The relevant data for free space for all the instruments is provided in Figure 12b. In addition we also show in Figure $12 \mathrm{~b}$ the dose measured by MSL-RAD ${ }_{B}$ during the cruise phase to Mars (December 2011 - July 2012).

Figure 12a clearly shows the variations of the dose rates over the solar cycle with maximum values at the end of 2009 and in 2019 (about $240 \mu \mathrm{Gy} / \mathrm{d}$ at the lunar surface and $110 \mu \mathrm{Gy} / \mathrm{d}$ in ISS orbit). The minimum of the GCR intensity during the investigated time period extended from about the middle of 2013 to the middle of 2015 (about $140 \mu \mathrm{Gy} / \mathrm{d}$, $120 \mu \mathrm{Gy} / \mathrm{d}$ and $90 \mu \mathrm{Gy} / \mathrm{d}$ at the Martian surface, the lunar 

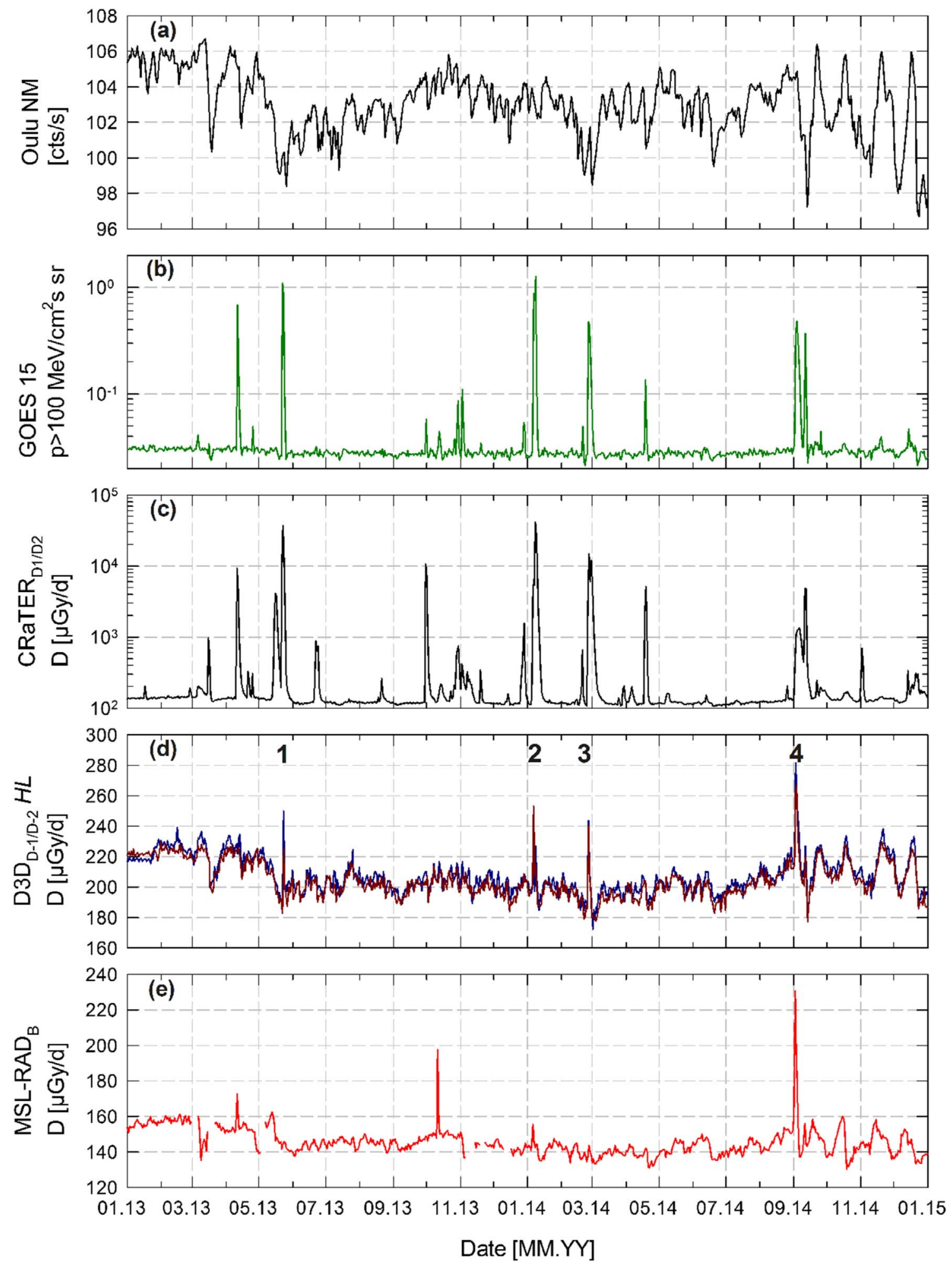

Fig. 10. (a) Oulu NM count rates; (b) GOES 15 proton flux > $100 \mathrm{MeV}$; (c) daily dose rates for CRaTER $\mathrm{D} 1 / \mathrm{D} 2_{2}$; (d) daily dose rates for $\mathrm{D} 3 \mathrm{D}_{\mathrm{D}-1}$ (dark blue) and $\mathrm{D}_{3} \mathrm{D}_{\mathrm{D}-2}$ (dark red) based on $H L$-value cut; Numbers (1-4) correspond to the events listed in Table 4; and (e) daily dose rates measured with the MSL-RAD $\mathrm{B}$ detector for the same time period. 
Table 4. Newly identified SPE events seen with the $\mathrm{D}_{\mathrm{D}} \mathrm{D}_{\mathrm{D}-1 / \mathrm{D}-2}$ instruments on board the ISS. These events have also been measured by AMS02, PAMELA, SOHO/EPHIN and $\mathrm{CRaTER}_{\mathrm{D} 1 / \mathrm{D} 2}$ as well as (for two events) with MSL-RAD $\mathrm{B}_{\mathrm{B}}$.

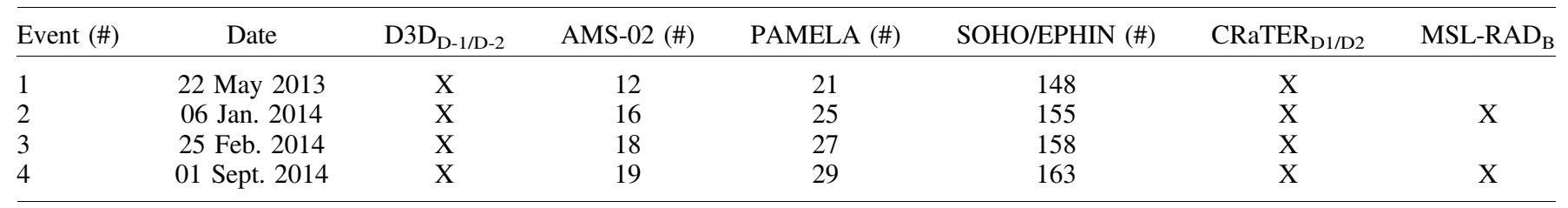

Note: AMS-02 data are provided from Bindi (2016) and her Table 1 listing the intense solar events between May 2011 and Feb 2014 associated with a proton increase observed by AMS-02 near $1 \mathrm{GV}$ and above. The relevant number of the events (\#) measured by AMS-02 from Table 1 is given in the AMS-02 column. PAMELA data are provided from Bruno et al. (2018) and their Table 1 listing the major SEP events observed by PAMELA between July 2006 and September 2014. The relevant number of the event (\#) measured by PAMELA from Table 1 is given in the PAMELA column. SOHO/EPHIN data are provided from Paassilta et al. (2017) and their Table 3. The relevant number of the events (\#) measured by SOHO/EPHIN from Table 3 is provided in the SOHO/EPHIN column.

Table 5. Dose rates for $L>4$ (pre-event and event) for the May 2013 and January, February and September 2014 SPEs.

\begin{tabular}{lccc}
\hline Date & \multicolumn{3}{c}{ Dose rate $(\mu \mathrm{Gy} / \mathrm{h})$ for $L>4$} \\
\cline { 2 - 3 } & Pre-event & Event & \\
\hline 22/23 May 2013 & $7.6 \pm 0.1$ & $9.9 \pm 0.1$ & 30.3 \\
05/06 Jan. 2014 & $8.4 \pm 0.1$ & $11.1 \pm 0.2$ & 32.3 \\
24/25 Feb. 2014 & $7.7 \pm 0.1$ & $10.2 \pm 0.1$ & 29.8 \\
01/02 Sept. 2014 & $8.9 \pm 0.1$ & $11.1 \pm 0.1$ & 25.4 \\
\hline
\end{tabular}
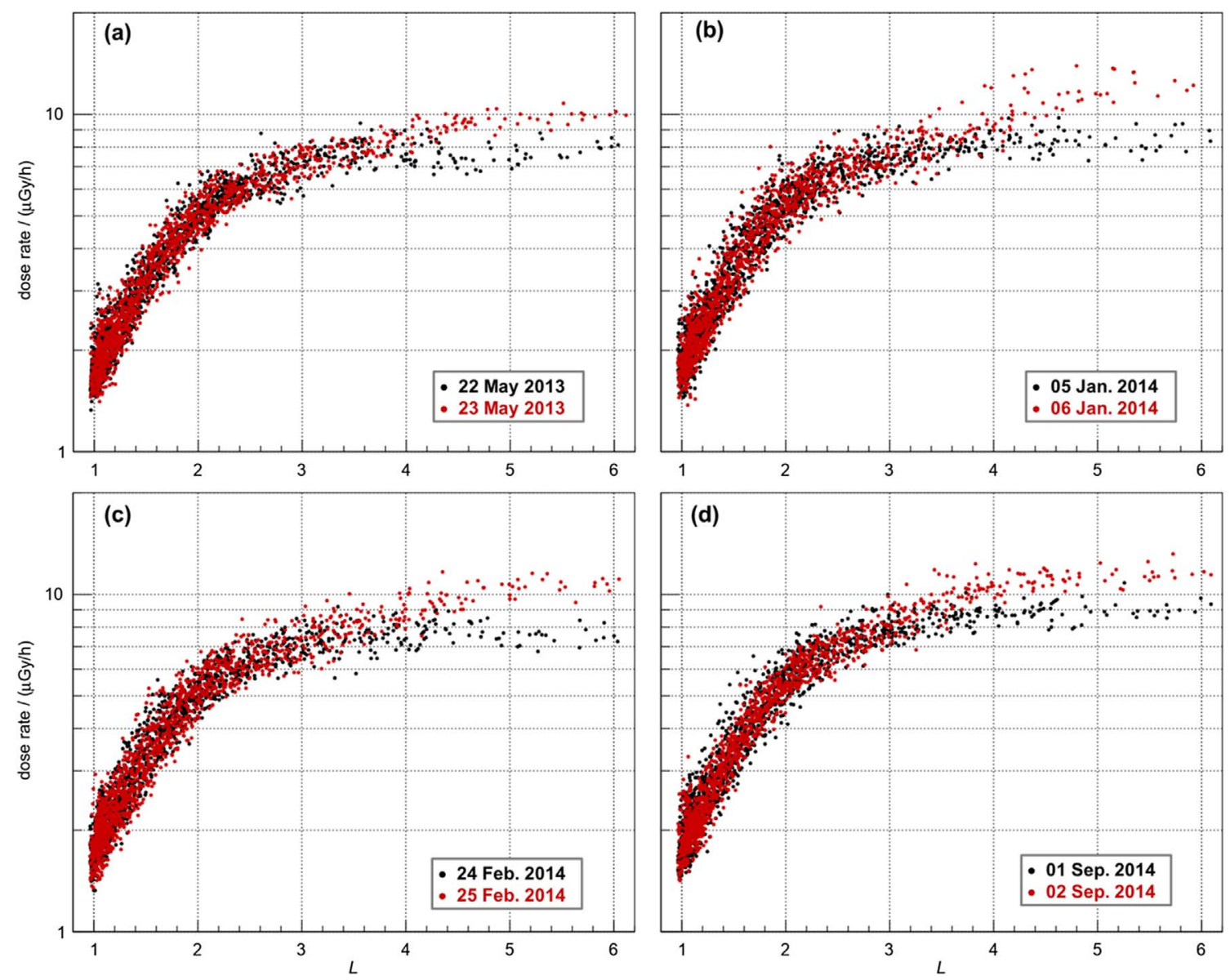

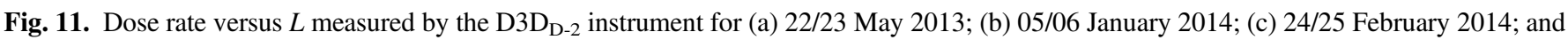
(d) 01/02 September 2014. Note: Pre-event data are given in black and event data in red (see also Table 5). 

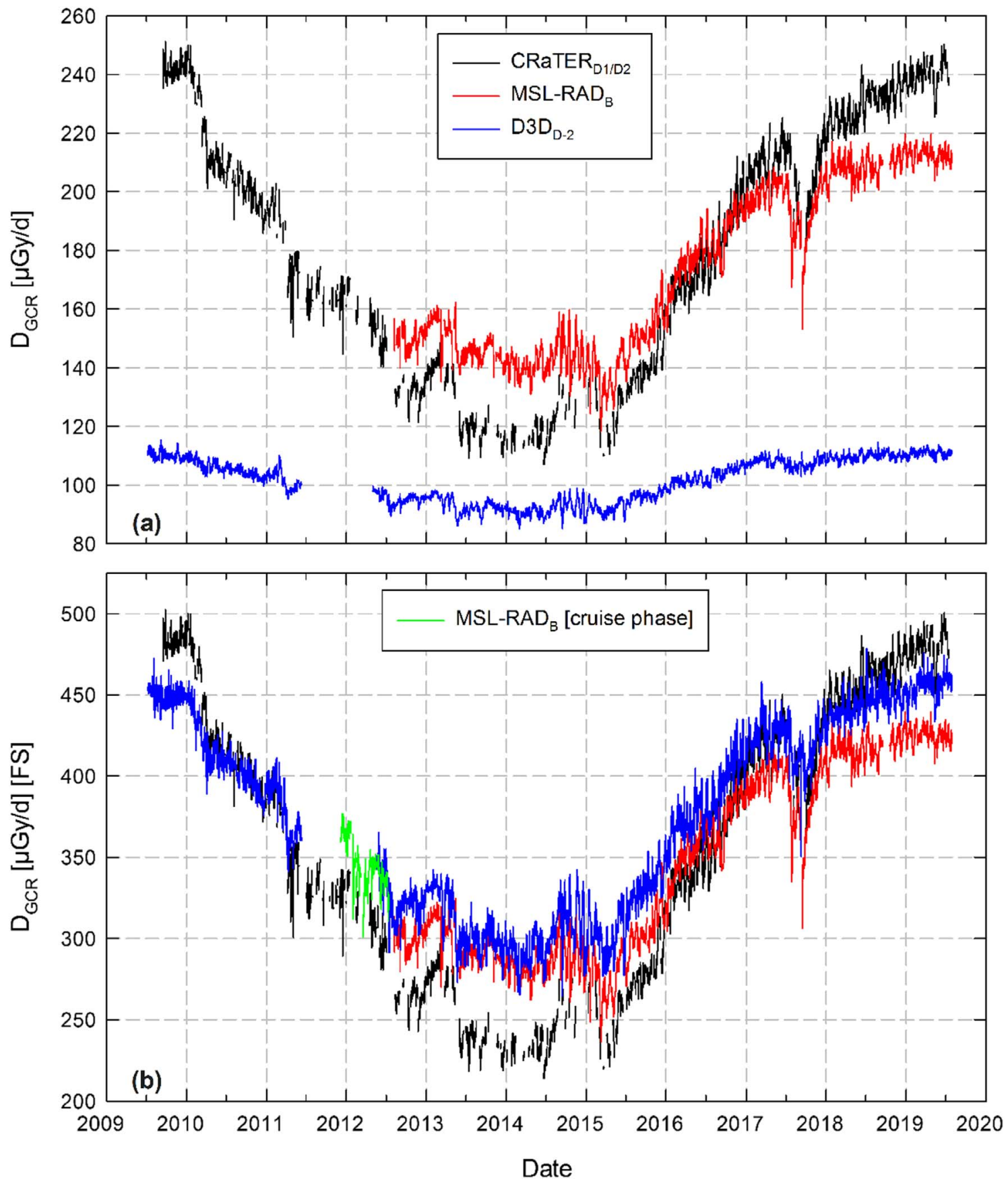

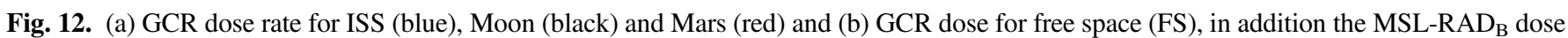
rate measured during the cruise phase to Mars (green) is provided. Note: all contributions from SPEs to the data of $\mathrm{D}^{2} \mathrm{D}_{\mathrm{D}-2}, \mathrm{CR}_{\mathrm{T}} \mathrm{TER}_{\mathrm{D} 1 / \mathrm{D} 2}$ and MSL-RAD ${ }_{\mathrm{B}}$ have been removed from (a) and (b).

surface and in the ISS orbit, respectively). During the GCR intensity maxima, the dose rate within the Columbus module in the ISS orbit is about a factor two lower than on the surface of Mars and Moon. The latter two are comparable in magnitude but the dose rate on Mars is less affected by modulation during the solar cycle, because the nearly-constant atmospheric shielding $\left(21 \pm 2 \mathrm{~g} / \mathrm{cm}^{2}\right)$ reduces the flux of the lower energetic GCR that are most affected by solar modulation. That is, RAD is shielded from the most variable components of the GCR. This effect also applies to some extent to the ISS data, since the Columbus module is moderately shielded. Of the three data sets reported here, CRaTER sees the largest variations since the D1/D2 detector pair is only very lightly shielded. The application of the above described procedures to the three data sets in order to estimate the dose rates in free space results in values which differ by about $25 \%$ during solar minimum and 

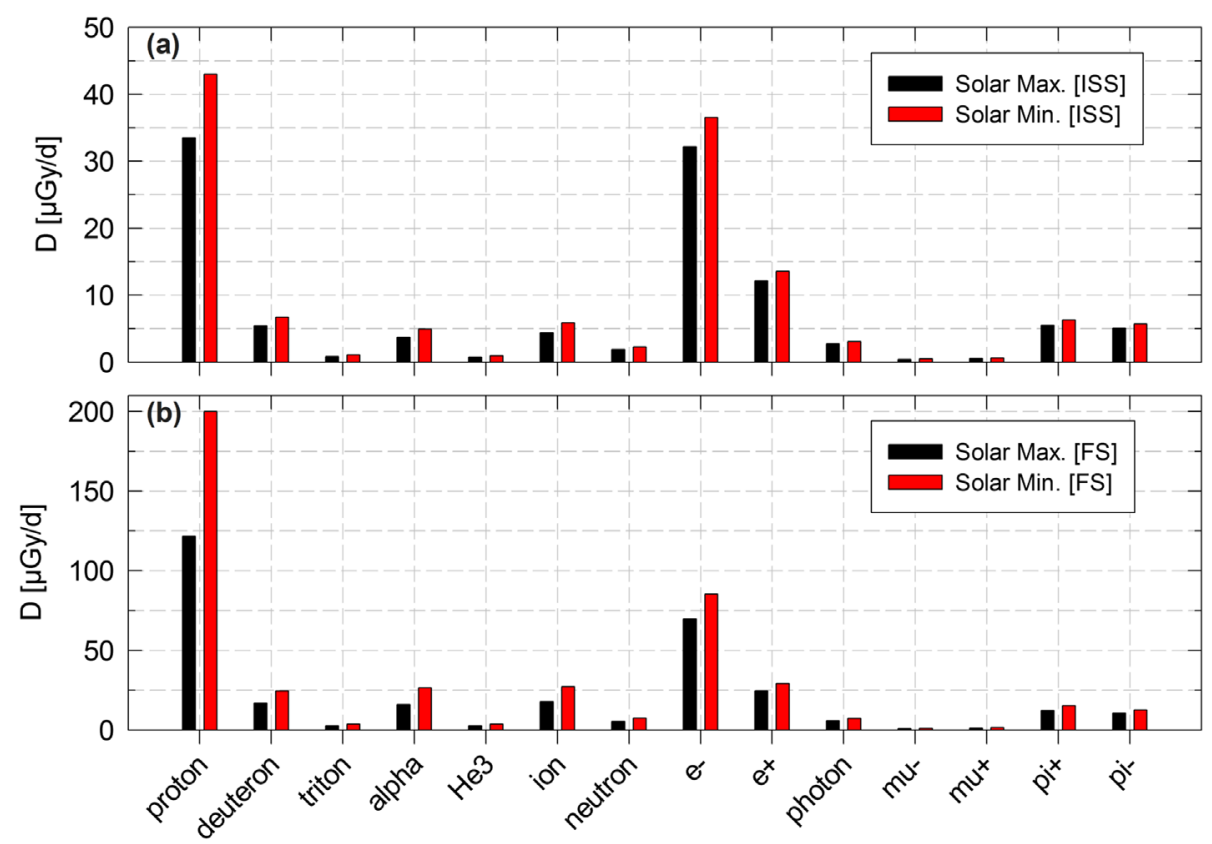

Fig. 13. Calculated contributions to the absorbed dose in Si by different particle species during solar maximum and solar minimum conditions (a) for ISS orbit and (b) for free space.

Table 6. Measured and calculated GCR dose values for solar maximum (March 2015) and solar minimum (Jan 2019) conditions for ISS orbits and for free space.

\begin{tabular}{|c|c|c|c|c|c|c|}
\hline \multirow[t]{3}{*}{ MM.YY } & \multicolumn{6}{|c|}{ Dose rate $(\mu \mathrm{Gy} / \mathrm{d})$} \\
\hline & $\mathrm{D} 3 \mathrm{D}_{\mathrm{D}-1} \mathrm{GCR}$ & $\mathrm{D} 3 \mathrm{D}_{\mathrm{D}-2} \mathrm{GCR}$ & GEANT4 & D3D $D_{D-1} G C R$ & $\mathrm{D} 3 \mathrm{D}_{\mathrm{D}-2} \mathrm{GCR}$ & GEANT4 \\
\hline & \multicolumn{3}{|c|}{ ISS } & \multicolumn{3}{|c|}{ Free space $(\mathrm{FS})$} \\
\hline 03.15 & 100.2 & 99.6 & 108.9 & 296 & 294 & 308 \\
\hline 01.19 & 123.1 & 121.9 & 131.0 & 457 & 450 & 455 \\
\hline
\end{tabular}

Note: The $\mathrm{D}_{3} \mathrm{D}_{\mathrm{D}-1}$ and $\mathrm{D} 3 \mathrm{D}_{\mathrm{D}-2}$ GCR dose values for the ISS orbits have been derived by using the DLR GCR/SAA separation procedure as described in Section 4.2.1.

maximum conditions and less during times of intermediate modulation (Fig. 12b). Being the least shielded of the three experiments, CRaTER data again shows the strongest modulation dependence and compared to the other experiments the dose rates for the free space estimate are lower during solar maximum conditions and greater during solar minimum conditions. This effect is caused by secondary particle production and scattering effects in the shielding of the Columbus module and the Martian regolith (albedo). The relative contribution of these secondary and albedo particles is increased during solar maximum conditions due to the increased fraction of primary particles with higher energy. The estimate for the free space dose rate extends from approximately $420 \mu \mathrm{Gy} / \mathrm{d}$ to $480 \mu \mathrm{Gy} / \mathrm{d}$ in 2019. For solar maximum conditions the estimate for the free space dose rate is reduced to approximately $240 \mu \mathrm{Gy} / \mathrm{d}$ and $300 \mu \mathrm{Gy} / \mathrm{d}$ which corresponds to a decrease of $40 \%$ compared to the GCR intensity maximum.

\subsection{GCR dose simulations (ISS and free space)}

The simulations for the GCR environment are based on two examples, namely the GCR dose for nominal ISS orbits and the
GCR dose for the ISS Free Space configuration. Both examples are calculated for a solar maximum condition (March 2015) and solar minimum condition (January 2019). The results of the measurements for March 2015 and January 2019 and the respective results of the GEANT4 calculations are provided in Table 6 for the ISS and the Free Space configuration. It can be seen that while the absorbed dose onboard the ISS increased by $\sim 22 \%$ from 2015 to 2019 the estimates for the dose rates in free space, due to the lack of the Earth's geomagnetic shielding increase by $\sim 54 \%$.

In addition, Figure 13 provides the particle contributions to the dose for the two scenarios and the two times in the solar cycle. Figure 13a shows the particle contribution for the ISS scenario, while Figure 13b provides data for the Free Space environment. It should be kept in mind that these values are the absorbed dose in $\mathrm{Si}$, and, as shown by Matthiä et al. (2018), they do not fully relate to relevant radiation protection quantities such as the effective dose equivalent, where the self-shielding of the body influences the relevant quantities. Additionally, silicon detectors have a low sensitivity to neutrons and the neutron dose to tissue or water in the identical radiation field is considerably higher. 


\section{Summary}

Within this work we have demonstrated two different ways to separate the GCR from the SAA contributions for instruments measuring the radiation environment inside the ISS. We have shown that the data measured on board the ISS for the GCR contribution to the absorbed dose can be used, based on relevant $L$-value cuts, as surrogate for a detector flying in a correspondingly shielded environment in free space. Further on the applied $L$-value cuts enabled us to identify four previously hidden SPEs seen with the $\mathrm{D} \mathrm{D}_{\mathrm{D}-1 / \mathrm{D}-2}$ instrument in the years 2013 and 2014 enabling us comparing them to data measured with the CRaTER and the MSL-RAD instruments. We have also shown the long term variation of the GCR environment starting from the deep solar minimum of cycle 23 in 2009 covering almost the full solar cycle 24 up to the now approaching minimum in 2020. GCR dose values have been shown for the ISS, the surface of the Moon, on the way to and on the surface of Mars, and these have been extrapolated to a free space environment thereby giving for the first time the full picture for data needed also for exploration human missions.

Acknowledgements. For DOSIS 3D CAU, Kiel, Germany was supported by DLR under Grants 50WB0826, 50WB1026, 50WB1232, and 50WB1533. At DLR, Cologne, DOSIS 3D was supported by the DLR grant FuE-Projekt "ISS LIFE", (Programm RF-FuW, Teilprogramm 475). For DOSIS 3D the authors gratefully acknowledge the support of ESA as well as CADMOS, France, OHB and DLR-MUSC, Germany. The MSL-RAD project is supported in the United States by the National Aeronautics and Space Administration's Human Exploration and Operations Mission Directorate, under Jet Propulsion Laboratory subcontract \#1273039 to Southwest Research Institute, and in Germany by the German Aerospace Center (DLR) and DLR's Space Administration grant numbers 50QM0501, 50QM1201, and 50QM1701 to the Christian Albrechts University, Kiel. The CRaTER team at the University of New Hampshire is supported by the NASA Lunar Reconnaissance Orbiter program, contract \#NNG11PA03C. DOSIS-3D-DOSTEL are available at: http://swe.ssa.esa.int/ human-space-flight. All data used here from the CRaTER and MSL-RAD experiments are available through the NASA Planetary Data System (PDS). Data sets are hosted on the University of California, Los Angeles Planetary Plasma Interactions (PPI) node, https://pds-ppi.igpp.ucla.edu. Data are available in both raw ("EDR") and human-readable ("RDR") formats. The authors would like to thank the Sodankyla Geophysical Observatory and the website teams (http://cosmicrays.oulu.fi) for providing the Oulu neutron monitor data. The authors would like to thank the Royal Observatory of Belgium, Brussels for the provision of the sunspot data (http://www.sidc.be/silso/). The authors would like to thank the HIMAC team at NIRS in Japan for providing lots of experiment time for the calibration of the instruments in the frame of various HIMAC Research Projects. The editor thanks two anonymous reviewers for their assistance in evaluating this paper.

\section{References}

Agostinelli S, Allison J, Amako KA, Apostolakis J, Araujo H, et al. 2003. GEANT4-a simulation toolkit. Nucl Instrum Meth A 506(3): 250-303. https://doi.org/10.1016/S0168-9002(03)01368-8.

Allison J, Amako K, Apostolakis JEA, Araujo HAAH, Dubois PA, et al. 2006. Geant 4 developments and applications. IEEE Trans Nucl Sci 53: 270-278. https://doi.org/10.1109/TNS.2006.869826.

Allison J, Amako K, Apostolakis J, Arce P, Asai M, et al. 2016. Recent developments in GEANT4. Nucl Instrum Meth A 835: 186-225. https://doi.org/10.1016/j.nima.2016.06.125.

Berger T, Przybyla B, Matthiä D, Reitz G, Burmeister S, et al. 2016. DOSIS \& DOSIS 3D: Long term dose monitoring on board the Columbus Laboratory of the International Space Station (ISS). J Space Weather Space Clim 6: A39. https://doi.org/10.1051/swsc/ 2016034.

Berger T, Burmeister S, Matthiä D, Przybyla B, Reitz G, et al. 2017. DOSIS \& DOSIS 3D: Radiation measurements with the DOSTEL instruments on board the Columbus Laboratory of the ISS in the years 2009-2016. J Space Weather Space Clim 7: A8. https://doi. org/10.1051/swsc/2017005.

Berger T, Matthiä D, Burmeister S, Rios R, Lee K, et al. 2018. The solar particle event on 10 September 2017 as observed on-board the International Space Station (ISS). Space Weather 16: 11731189. https://doi.org/10.1029/2018SW001920.

Berrilli F, Casolino M, Del Moro D, Di Fino L, Larosa M, et al. 2014. The relativistic solar particle event of May 17th, 2012 observed on board the International Space Station. J Space Weather Space Clim 4: A16. https://doi.org/10.1051/swsc/2014014.

Bindi V. 2016. Solar energetic particles measured by AMS-02. In: The 34th International Cosmic Ray Conference, 30 July-6 August, 2015, The Hague, The Netherlands. https://doi.org/10.22323/1.236.0108.

Bruno A, Bazilevskaya GA, Boezio M, Christian ER, de Nolfo GA. 2018. Solar energetic particle events observed by the PAMELA mission. ApJ 862: 97. https://doi.org/10.3847/1538-4357/aacc26.

Cohen CMS, Mewaldt RA. 2018. The ground-level enhancement event of September 2017 and other large solar energetic particle events of cycle 24. Space Weather 16: 1616-1623. https://doi.org/ 10.1029/2018SW002006.

Durante M, Cucinotta FA. 2011. Physical basis of radiation protection in space travel. Rev Mod Phys 83(4): 1245-1281. https://doi.org/10.1103/RevModPhys.83.1245.

Ehresmann B, Zeitlin C, Hassler DM, Wimmer-Schweingruber RF, Böhm E, et al. 2014. Charged particle spectra obtained with the Mars Science Laboratory Radiation Assessment Detector (MSL/RAD) on the surface of Mars. J Geophys Res Planets 119: 468-479. https://doi.org/10.1002/2013JE004547.

Ehresmann B, Hassler DM, Zeitlin C, Guo J, Köhler J, et al. 2016. Charged particle spectra measured during the transit to Mars with the Mars Science Laboratory Radiation Assessment Detector (MSL/RAD). Life Sci Space Res 10: 29-37. https://doi.org/ 10.1016/j.1ssr.2016.07.001.

Ehresmann B, Hassler DM, Zeitlin C, Guo J, WimmerSchweingruber RF, et al. 2018. Energetic particle radiation environment observed by RAD on the surface of Mars during the September 2017 event. Geophys Res Lett 45: 5305-5311. https://doi.org/10.1029/2018GL077801.

Geant4-Collaboration. 2016. Physics reference manual - version: Geant4 10.3 (9 December 2016). http://geant4-userdoc.web.cern. ch/geant4-userdoc/UsersGuides/PhysicsReferenceManual/fo/PhysicsReferenceManual.pdf. 
Hassler DM, Zeitlin C, Wimmer-Schweingruber RF, Böttcher S, Martin C, et al. 2012. The radiation assessment detector (RAD) investigation. Space Sci Rev 170(1-4): 503-558. https://doi.org/ 10.1007/s11214-012-9913-1.

Hassler DM, Zeitlin C, Wimmer-Schweingruber RF, Ehresmann B, Rafkin S, et al. 2014. Mars surface radiation environment measured with the mars science laboratory's curiosity rover. Science $\mathbf{3 4 3}$ (6169): 1244797. https://doi.org/10.1126/science. 1244797.

Hassler DM, Zeitlin C, Ehresmann B, Wimmer-Schweingruber RF, Guo J, et al. 2018. Space Weather on the surface of Mars: Impact of the September 2017 events. Space Weather 16: 1702-1708. https://doi.org/10.1029/2018SW001959.

ISO. 2004. ISO 15390:2004 space environment (natural and artificial) - Galactic cosmic ray model. https://www.iso.org/standard/37095.html.

Jiggens P, Clavie C, Evans H, O'Brien TP, Witasse O, et al. 2018. In-situ data and effect correlation during September 2017 solar particle event. Space Weather 17: 99-117. https://doi.org/10.1029/ 2018SW001936.

Köhler J, Ehresmann B, Zeitlin C, Wimmer-Schweingruber RF, Hassler DM, et al. 2015. Measurements of the neutron spectrum in transit to Mars on the Mars Science Laboratory. Life Sci Space Res 5: 6-12. https://doi.org/10.1016/j.lssr.2015.03.001.

Matthiä D, Berger T, Mrigakshi AI, Reitz G. 2013. A ready-to-use galactic cosmic ray model. Adv Space Res 51: 329-338. https://doi.org/10.1016/j.asr.2012.09.022.

Matthiä D, Meier MM, Berger T. 2018. The solar particle event on 10-13 September 2017 - spectral reconstruction and calculation of the radiation exposure in aviation and space. Space Weather 16: 977-986. https://doi.org/10.1029/2018SW001921.

Mcllwain CE. 1961. Coordinates for mapping the distribution of magnetically trapped particles. J Geophys Res 66(11): 3681-3691. https://doi.org/10.1029/JZ066i011p03681.

Mishev A, Poluianov S, Usoskin I. 2017. Assessment of spectral and angular characteristics of sub-GLE events using the global neutron monitor network. J Space Weather Space Clim 7: A28. https://doi. org/10.1051/swsc/2017026.

Mitrofanov IG, Litvak ML, Varenikov AB, Barmakov YN, et al. 2012. Dynamic Albedo of Neutrons (DAN) experiment on board NASA's Mars Science Laboratory. Space Sci Rev 170(1-4): 559-582. https://doi.org/10.1007/s11214-012-9924-y.

Narici L, Berger T, Burmeister S, Di Fino L, Rizzo A, et al. 2017. Exploiting different active silicon detectors in the International Space Station: ALTEA and DOSTEL galactic cosmic radiation (GCR) measurements. J Space Weather Space Clim 7: A18. https://doi.org/10.1051/swsc/2017016.

Norbury J, Whitman WK, Lee K, Slaba TC, Badavi FF. 2018. Comparison of space radiation GCR models to recent AMS data. Life Sci Space Res 18: 64-71. https://doi.org/10.1016/j.lssr.2018. 05.003.

Paassilta M, Raukunen O, Vainio R, Valtonen E, Papaioannou A, et al. 2017. Catalogue of $55-80 \mathrm{MeV}$ solar proton events extending through solar cycles 23 and 24. J Space Weather Space Clim 7: A14. https://doi.org/10.1051/swsc/2017013.

Schwadron NA, Rahmanifard F, Wilson J, Jordan AP, Spence HE, Joyce CJ, et al. 2018. Update on the worsening particle radiation environment observed by CRaTER and implications for future human deep-space exploration. Space Weather 16: 289-303. https://doi.org/10.1002/2017SW001803.
Smart DF, Shea MA. 2005. A review of geomagnetic cutoff rigidities for earth orbiting spacecraft. Adv Space Res 36(10): 2012-2020. https://doi.org/10.1016/j.asr.2004.09.015.

Spence HE, Case AW, Golightly MJ, Heine T, Larsen BA, et al. 2010. CRaTER: The cosmic ray telescope for the effects of radiation experiment on the lunar reconnaissance orbiter mission. Space Sci Rev 150(1-4): 243-284. https://doi.org/10.1007/s11214009-9584-8.

Spence HE, Golightly MJ, Joyce CJ, Looper MD, Schwadron NA, et al. 2013. Relative contributions of galactic cosmic rays and lunar proton "albedo" to dose and dose rates near the Moon. Space Weather 11: 643-650. https://doi.org/10.1002/2013SW000995.

Thakur N, Gopalswamy N, Xie H, Mäkelä P, Yashiro S, et al. 2014. Ground level enhancement in the 2014 January 6 solar energetic particle event. ApJL 790: L13. https://doi.org/10.1088/2041-8205/ 790/1/L13.

Thébault E, Finlay CC, Beggan CD, Alken P, Aubert J, et al. 2015. International geomagnetic reference field: The 12th generation. Earth Planets Space 67(1): 79. https://doi.org/10.1186/s40623015-0228-9.

Tsyganenko N. 1989. A magnetospheric magnetic field model with a warped tail current sheet. Planet Space Sci 37(1): 5-20. https://doi. org/10.1016/0032-0633(89)90066-4.

Usoskin IG, Ibragimov A, Shea MA, Smart DF. 2016. Database of ground level enhancements (GLE) of high energy solar proton events. In: The 34th International Cosmic Ray Conference, 30 July-6 August, 2015, The Hague, The Netherlands. https://doi.org/ 10.22323/1.236.0054.

Whitman K, Norbury JW, Lee K, Slaba TC, Badavi FF. 2019. Comparison of space radiation GCR models to AMS heavy ion data. Life Sci Space Res 22: 76-88. https://doi.org/10.1016/j. 1ssr.2019.07.007.

Wimmer-Schweingruber RF, Köhler J, Hassler DM, Guo J, Appel JK, et al. 2015. On determining the zenith angle dependence of the Martian radiation environment at Gale Crater altitudes. Geophys Res Lett 42: 10557-10564. https://doi.org/10.1002/2015GL066664.

Zeitlin C, Hassler D, Cucinotta F, Ehresmann B, WimmerSchweingruber RF, et al. 2013. Measurements of energetic particle radiation in transit to Mars on the Mars Science Laboratory. Science 340(6136): 1080-1084. https://doi.org/10.1126/science.1235989.

Zeitlin C, Hassler DM, Wimmer-Schweingruber RF, Ehresmann B, Appel J, et al. 2016. Calibration and characterization of the radiation assessment detector (RAD) on curiosity. Space Sci Rev 201(1-4): 201-233. https://doi.org/10.1007/s11214-016-0303-y.

Zeitlin C, Hassler DM, Guo J, Ehresmann B, Wimmer-Schweingruber RF, et al. 2018. Investigation of the radiation hazard observed by RAD on the Surface of Mars during the Sept 2017 solar particle event. Geophys Res Lett 45: 5845-5851. https://doi. org/10.1029/2018GL077760.

Zeitlin C, Schwadron NA, Spence HE, Jordan AP, Looper MD, Wilson J, et al. 2019a. Update on galactic cosmic ray integral flux measurements in lunar orbit with CRaTER. Space Weather 17: 1011-1017. https://doi.org/10.1029/2019SW002223.

Zeitlin C, Narici L, Rios RR, Rizzo A, Stoffle N, et al. 2019b. Comparisons of high-linear energy transfer spectra on the ISS and in deep space. Space Weather 17: 396-418. https://doi.org/ $10.1029 / 2018$ SW002103.

Cite this article as: Berger T, Matthiä D, Burmeister S, Zeitlin C, Rios R, et al. 2020. Long term variations of galactic cosmic radiation on board the International Space Station, on the Moon and on the surface of Mars. J. Space Weather Space Clim. 10, 34. 